\title{
Electron transport and energy relaxation in dilute magnetic alloys
}

\author{
M. G. Vavilov, ${ }^{1}$ L. I. Glazman, ${ }^{1}$ and A. I. Larkin ${ }^{1,2}$ \\ 1 Theoretical Physics Institute, University of Minnesota, Minneapolis, MN 55455 \\ 2 Landau Institute for Theoretical Physics, Moscow, 117940 Russia
}

(Dated: May 9, 2003)

\begin{abstract}
We consider the effect of the RKKY interaction between magnetic impurities on the electron relaxation rates in a normal metal. The interplay between the RKKY interaction and the Kondo effect may result in a non-monotonic temperature dependence of the electron momentum relaxation rate, which determines the Drude conductivity. The electron phase relaxation rate, which determines the magnitude of the weak localization correction to the resistivity, is also a non-monotonic function of temperature. For this function, we find the dependence of the position of its maximum on the concentration of magnetic impurities. We also relate the electron energy relaxation rate to the excitation spectrum of the system of magnetic impurities. The energy relaxation determines the distribution function for the out-of-equilibrium electrons. Measurement of the electron distribution function thus may provide information about the excitations in the spin glass phase.
\end{abstract}

PACS numbers: 73.23.-b, 75.10.Nr, 72.10.Fk, 71.10.Ay

\section{INTRODUCTION}

Electron transport in normal metals is known to be very sensitive to the presence of magnetic impurities in a metal. Scattering of conduction electrons off such impurities scrambles the electron spin. A tiny concentration of magnetic impurities results in an observable effect low-temperature saturation of the phase relaxation rate ${ }^{\frac{1}{1}}$. Magnetic impurities apparently also facilitate the energy transfer between electrons ${ }^{2.3}$. At higher concentrations ${ }^{1}$, a minimum in the temperature dependence of the resistivity becomes evident, which is a manifestation of the Kondo effect $\stackrel{4}{*}$. These three observations fit very well with a picture of uncorrelated magnetic impurities.

Investigation of the resistivity at even higher concentration of magnetic impurities, reveals deviations from the picture of uncorrelated localized magnetic moments. The temperature dependence of the resistivity, in addition to the aforementioned minimum, develops a maximum ${ }^{5.6 .7 .8 .9}$ at lower temperatures. The electron phase relaxation rate was recently measured ${ }^{10.11}$ on AuFe alloys with magnetic impurity (Fe) concentration ranging from 7.1 to $60 \mathrm{ppm}$. At the upper end of this range, correlations between the localized spins may become important, as evidenced by the temperature dependence of the resistivity ${ }^{5.11}$.

In this paper we investigate the effect of correlation between spins of magnetic impurities on the electronic transport properties of a metal. Specifically, we study the Kondo contribution to the Drude resistivity, the weaklocalization correction to the conductivity, and the electron energy relaxation rate.

The leading mechanism of correlations is known to be the Ruderman-Kittel-Kasuya-Yosida (RKKY) interaction between impurities. The interaction lifts the degeneracy in the excitation spectrum of impurities. In this respect, the effect of RKKY interaction is somewhat similar to the effect of an external magnetic field which causes Zeeman splitting of the spin states. It is known that the magnetic field reduces the electron relaxation rates at low energies 3.12 .13 .14 , and also suppresses the Kondo effect ${ }^{15}$. Unlike the uniform Zeeman splitting, however, interaction between the spins results in a broad spectrum of energies of collective spin states. Therefore, the quantitative manifestations of the RKKY interaction in the electron transport are different from that of the Zeeman energy.

The difficulty of the low-temperature electron transport problem is associated with the complexity of the spin glass state. In this paper, we study in detail the transport at relatively high temperatures (or electron energy transfers, in the case of energy relaxation). We perform analytical calculation using the method of the virial expansion ${ }^{16.17}$ in the RKKY interaction between magnetic impurities. The method is based on the following concept. Since the RKKY interaction decreases fast as a function of the distance between impurities, the impurities have to be close to each other for the interaction between them to compete with thermal smearing and to affect transport properties of conduction electrons. We perform the virial expansion to the second order in the density of magnetic impurities, which corresponds to accounting for the interaction within impurity pairs.

Electron scattering off magnetic impurities contributes to the temperature dependence of the resistivity: due to the Kondo effect, the resistivity increases as the temperature is lowered. Similar to the Zeeman splitting, the RKKY interaction between magnetic impurities may stop the development of the Kondo effect. The interplay between the RKKY interaction and the Kondo effect leads to a maximum in the temperature dependence of the resistivity ${ }^{7.8 .9}$ If the characteristic temperature of the spin glass formation exceeds significantly the Kondo temperature (high concentration of magnetic impurities $n_{\mathrm{s}}$ ), then the resistivity has a maximum at a temperature in the region of applicability of the virial expansion. On the contrary, at small $n_{\mathrm{s}}$ the maximum of resistivity occurs at zero temperature. 
Magnetic impurities also affect the magneto-resistance in weak magnetic fields. This magneto-resistance is due to the weak localization (WL) effect ${ }^{18.19}$ Being an interference phenomenon, the WL is limited by the electron phase relaxation. We calculate the phase relaxation rate taking into account the RKKY interaction between magnetic impurities. At high impurity concentration, the phase relaxation rate has a maximum, similar to the maximum in the resistivity. At low concentration, unlike the Drude resistivity, the phase relaxation retains a maximum at a finite temperature (of the order of the Kondo temperature).

The RKKY interaction lifts the degeneracy of the magnetic impurity states. Therefore an out-of-equilibrium electron may loose its energy by exciting the impurity spin degrees of freedom. The corresponding relaxation rate is a function of the transferred energy. This rate provides information about the spin excitations spectrum. We derive the corresponding kinetic equation for the electron distribution function. We also make specific predictions for the relaxation rate at sufficiently large energy transfers, which may be accounted for by the virial expansion.

Before we proceed, we emphasize that in this paper we consider the effect of the interaction between Kondo impurities only on kinetic properties (conductance, energy relaxation) of electrons in a metal. The interaction between magnetic impurities may also affect thermodynamic properties (such as heat capacity, susceptibility, superconducting transition temperature) of conduction electrons $\frac{16.17 .20 .21}{}$ An analysis of the thermodynamic properties of electrons in dilute Kondo alloys was performed earlier, see e.g. ref. 20.

The paper is organized as follows. In the next section we introduce the model and discuss the effect of interaction between magnetic impurities on a spin correlation function. In Sec. III we perform calculations of the resistivity correction due to the electron scattering off magnetic impurities. Section IV contains analysis of the WL correction to the conductivity. In Sec. V we derive the kinetic equation for the system of magnetic impurities and conduction electrons and discuss the energy exchange rate between these two subsystems. Section VI contains discussions and conclusions.

\section{MODEL}

The scattering of conduction electrons off a magnetic impurity is described by the following Hamiltonian

$$
\hat{H}_{\mathrm{e}}=\mathcal{J} \hat{\boldsymbol{S}} \hat{\boldsymbol{\sigma}}
$$

where $\hat{\boldsymbol{S}}$ is the spin operator of a magnetic impurity and $\hat{\boldsymbol{\sigma}}$ is the spin operator of a conduction electron represented in terms of the Pauli matrices $\left\{\hat{\sigma}_{x}, \hat{\sigma}_{y}, \hat{\sigma}_{z}\right\}$. The exchange constant $\mathcal{J}$ is renormalized due to the Kondo effect and varies as a logarithmic function of energy $\varepsilon$ of conduction electrons. At temperature $T$ higher than the Kondo temperature $T_{\mathrm{K}}$, the exchange constant for thermal electrons, $\varepsilon \sim T$, is given by

$$
\mathcal{J}=\frac{2}{\nu} \ln ^{-1} \frac{T}{T_{\mathrm{K}}}, \quad T \gg T_{\mathrm{K}},
$$

where $\nu$ is the Fermi density of states per spin degree of freedom.

In order to evaluate the effects of electron scattering off magnetic impurities on electron transport, we introduce the statistical averages of impurity spin components, $\left\langle\hat{S}_{\alpha} \hat{S}_{\beta}\right\rangle$, with respect to thermodynamic states of the magnetic impurity system. We show that the electron transport properties in a metal with strong spin-orbit coupling are determined by the following spin correlator

$$
K(t)=\langle\hat{\boldsymbol{S}}(0) \hat{\boldsymbol{S}}(t)\rangle .
$$

In general, the correlation function $K(t)$ can be rewritten in terms of exact quantum states $|\xi\rangle$ of the system of magnetic impurities:

$$
K(\omega)=2 \pi \sum_{\xi \xi^{\prime}} \rho_{\xi}\left|\left\langle\xi|\boldsymbol{S}| \xi^{\prime}\right\rangle\right|^{2} \delta\left(E_{\xi}-E_{\xi^{\prime}}-\omega\right) .
$$

Here $E_{\xi}$ is the energy of state $|\xi\rangle$, and $\rho_{\xi}$ is the density matrix $\rho_{\xi} \propto \exp \left(-E_{\xi} / T\right)$.

For a free magnetic impurity with spin $S$ (all spin states are degenerate) the spin correlation function $K(t)$ does not depend on time and its Fourier transform has the form:

$$
K_{1}(\omega)=2 \pi S(S+1) \delta(\omega) .
$$

Using the Fermi golden rule and Eq. (5), we obtain the following expression for the electron scattering rate off magnetic impurities

$$
\frac{1}{\tau_{\mathrm{s}}}=2 \pi \nu n_{\mathrm{s}} \mathcal{J}^{2} S(S+1) .
$$

Here $n_{\mathrm{s}}$ is the magnetic impurity concentration per volume. The quantity $\tau_{\mathrm{s}}$ is the mean free time of scattering off magnetic impurities.

In metals, the leading interaction between magnetic moments of impurities is described by the RudermanKittel-Kasuya-Yosida (RKKY) mechanism. The corresponding Hamiltonian has the form:

$$
\hat{H}_{\mathrm{RKKY}}=\sum_{i j} V\left(r_{i j}\right) \hat{\boldsymbol{S}}_{i} \hat{\boldsymbol{S}}_{j}
$$

The magnitude of the RKKY interaction is given by the following expression

$$
V(r)=\frac{V_{0}(r)}{r^{3}} \cos \varphi,
$$

where $\varphi$ changes fast on the length scale of the Fermi wavelength $\lambda_{\mathrm{F}}$. The interaction constant $V_{0}$ may be represented in terms of the exchange constant and electron density of states $\nu$ :

$$
V_{0}(r)=\frac{\nu \mathcal{J}_{0}^{2}(r)}{2 \pi}, \quad \mathcal{J}_{0}(r)=\frac{2}{\nu} \frac{1}{\ln \left[v_{\mathrm{F}} /\left(r T_{\mathrm{K}}\right)\right]} .
$$


The typical value of the RKKY interaction between two impurities separated by distance $1 / n_{\mathrm{s}}^{1 / 3}$ (average distance between impurities) is

$$
T_{\mathrm{sg}}=n_{\mathrm{s}} V_{0}\left(r=n_{\mathrm{s}}^{-1 / 3}\right) \simeq \frac{2 n_{\mathrm{s}}}{\pi \nu \ln ^{2}\left(v_{\mathrm{F}} n_{\mathrm{s}}^{1 / 3} / T_{\mathrm{K}}\right)} .
$$

Due to the randomness of the RKKY Hamiltonian $\hat{H}_{\mathrm{RKKY}}$, finding of states $|\xi\rangle$ and corresponding energies $E_{\xi}$ is a hardly possible task. At sufficiently low temperature a phase transition into a spin glass state may occur with extremely complicated structure of the wave functions $|\xi\rangle$ and energy spectrum ${ }^{22}$ A transition temperature is comparable with the typical energy of the interaction between magnetic impurities $T_{\mathrm{sg}}$.

We focus our attention on the high temperature limit $T \gg T_{\mathrm{sg}}$ and calculate the spin-spin correlation function $K(t)$, Eq. (3), using the virial expansion method. In this method the interaction between magnetic impurities is taken into account only if the splitting of the spin states due to the interaction exceeds the system temperature; otherwise the interaction does not significantly change the spin correlation function. Therefore, the interaction is important only for magnetic impurities in clusters of the size $\sim \sqrt[3]{V_{0} / T}$. For a uniform distribution of magnetic impurities in the metal, the probability for a formation of such a cluster of $k$ impurities scales as $\left(T_{\mathrm{sg}} / T\right)^{k-1}$. We consider only clusters containing two $(k=2)$ magnetic impurities.

The energy states $|\xi\rangle$ of two interacting spins are classified by the total spin $J(J=0,1, \ldots, 2 S)$ and its projection $M$ on a fixed direction: $|\xi\rangle=|J, M\rangle$. The energy spectrum is given by

$$
E_{J}=V(r) \frac{J(J+1)-2 S(S+1)}{2} \equiv V(r) \epsilon_{J}
$$

and is degenerate with respect to the projection $M$. The spacing between levels with different $J$ is proportional to the magnitude of the RKKY interaction, Eq. 8

According to Eq. (4), the corresponding spin correlation function of a magnetic impurity within distance $r$ from another magnetic impurity may be represented in the form

$$
K_{2}(t, V)=\sum_{J, J^{\prime}=0}^{2 S} A_{J J^{\prime}} \frac{2 J+1}{Z(V)} e^{V\left\{i\left(\epsilon_{J}-\epsilon_{J^{\prime}}\right) t-\epsilon_{J} / T\right\}},
$$

where the statistical sum $Z(V)$ is

$$
Z(V)=\sum_{J=0}^{2 S}(2 J+1) e^{-V \epsilon_{J} / T},
$$

and the matrix elements $A_{J J^{\prime}}$ are

$$
A_{J J^{\prime}}=\sum_{M^{\prime}}\left\langle J M|\hat{\boldsymbol{S}}| J^{\prime} M^{\prime}\right\rangle\left\langle J^{\prime} M^{\prime}|\hat{\boldsymbol{S}}| J M\right\rangle .
$$

The analytical form of the matrix elements is presented in Appendix A. We emphasize that $A_{J J^{\prime}}$ are independent of the pair spin projection $M$.
As we will see in the following sections, the electron transport properties are determined by the spin correlation function $\overline{K(\omega)}$, averaged over configurations of magnetic impurities. We calculate $\overline{K(\omega)}$ within the virial approximation:

$$
\overline{K(\omega)}=K_{1}(\omega)+\int p(r)\left[K_{2}(\omega, V(r))-K_{1}(\omega)\right] d^{3} \boldsymbol{r},
$$

where $p(r)$ is the probability density for two magnetic impurities to be at distance $r$; for uniform impurity distribution $p(r)=n_{\mathrm{s}}$. Averaging over the relative position of two magnetic impurities in Eq. (14) can be performed in two steps. First, we make the substitution $r^{3}=V_{0} \cos \varphi /(T y)$, and then we perform integration over the fast varying phase $\varphi$. As the result, the spin correlator has the form 17

$$
\overline{K(\omega)}=K_{1}(\omega)+\delta \overline{K_{2}(\omega)},
$$

where

$$
\begin{aligned}
\delta \overline{K_{2}(\omega)}= & \frac{8 \pi}{3} \frac{T_{\mathrm{sg}} S(S+1)}{T} \int_{-\infty}^{+\infty} \frac{d y}{y^{2}}[P(\omega, y)-\delta(\omega)] \\
P(\omega, y)= & \sum_{J J^{\prime}=0}^{2 S} A_{J J^{\prime}} \frac{2 J+1}{S(S+1)} \frac{e^{-y \epsilon_{J}}}{Z(T y)} \\
& \times \delta\left(\omega-T y\left[\epsilon_{J}-\epsilon_{J^{\prime}}\right]\right) .
\end{aligned}
$$

We notice that the spin correlation function Eq. (15) increases as the frequency $\omega$ decreases:

$$
\begin{aligned}
\overline{K(\omega)}= & \frac{8 \pi}{3} \frac{T_{\mathrm{sg}}}{T} \sum_{J \neq J^{\prime}}^{2 S}(2 J+1) A_{J J^{\prime}} \frac{\left|\epsilon_{J}-\epsilon_{J^{\prime}}\right|}{\omega^{2}} \\
& \times \frac{\exp \left(-\frac{\omega \epsilon_{J}}{\left(\epsilon_{J}-\epsilon_{J^{\prime}}\right) T}\right)}{Z\left(\omega /\left(\epsilon_{J}-\epsilon_{J^{\prime}}\right)\right)}, \omega \neq 0 .
\end{aligned}
$$

One may expect that due to the $1 / \omega^{2}$ behavior of the spin correlation function, Eq. (17), the virial approximation breaks down even at $T \gtrsim T_{\mathrm{sg}}$. Nevertheless, due to the property $P(\omega, 0)=\delta(\omega)$, see Eq. (A4), the integrand in Eq. 16a has no singularity at $y=0$, and the slow modes $(\omega \lesssim T)$ of the spin system do not affect electron transport.

Equation (15) supplemented with Eqs. (13) and (16) determines the spin correlation function at high temperature $T \gg T_{\mathrm{sg}}$. Below we use these equations to describe the effect of interaction between magnetic impurities on electron transport in metals.

\section{RESISTIVITY}

The conductivity of a metal with isotropic impurities may be calculated according to the standard rules of the 
diagrammatic technique. Disregarding the interference corrections we have

$$
\sigma=\frac{e^{2} v_{\mathrm{F}}^{2}}{6} \int \frac{\overline{G^{R}}(\varepsilon, \mathbf{p}) \overline{G^{A}}(\varepsilon, \mathbf{p})}{T \cosh ^{2} \varepsilon / 2 T} \frac{d \varepsilon d \mathbf{p}}{(2 \pi)^{4}} .
$$

Here

$$
\overline{G^{R, A}}(\varepsilon, \mathbf{p})=\frac{1}{\varepsilon-\xi(\mathbf{p})-\Sigma^{R, A}(\varepsilon)}
$$

is the retarded or advanced Green function averaged over disorder, $\xi(\mathbf{p})=v_{\mathrm{F}}\left(|\mathbf{p}|-p_{\mathrm{F}}\right)$ is the electron energy, counted from the Fermi energy, $\Sigma^{R, A}(\varepsilon)$ is the electron retarded or advanced self energy, and $v_{\mathrm{F}}$ is the Fermi velocity. Performing the integration over momentum $\mathbf{p}$, we obtain

$$
\sigma=e^{2} \nu \frac{v_{\mathrm{F}}^{2}}{3} \int \frac{1}{\operatorname{Im} \Sigma^{A}(\varepsilon)} \frac{d \varepsilon}{4 T \cosh ^{2} \varepsilon / 2 T}
$$

with $\nu$ being the density of states of conduction electrons per one spin orientation.

We assume that the electron self energy part contains two components:

$$
\operatorname{Im} \Sigma^{A}(\varepsilon)=\frac{1}{2 \tau_{\mathrm{e}}}+n_{\mathrm{s}} \operatorname{Im} \overline{T(\varepsilon)}
$$

The first term in Eq. (21), $1 / 2 \tau_{\mathrm{e}}$, represents the effect of elastic scattering off non-magnetic impurities with $\tau_{\mathrm{e}}$ being the mean free elastic time. The second term, $n_{\mathrm{s}} \operatorname{Im} \overline{T(\varepsilon)}$, represents the effect of scattering of electrons with energy $\varepsilon$ off magnetic impurities. Here the scattering off a particular magnetic impurity is characterized by the $T$-matrix $T(\varepsilon)$; the self energy $\Sigma^{R}(\varepsilon)$ contains $\overline{T(\varepsilon)}$, averaged over various impurities.

Using the simple relation $\rho=1 / \sigma$ between the conductivity $\sigma$ and the resistivity $\rho$ and Eqs. (20) and (21), we represent the resistivity as a sum of two terms

$$
\rho=\rho_{\mathrm{e}}+\Delta \rho_{\mathrm{K}}
$$

The first term is the resistivity of a metal without magnetic impurities $\left(n_{\mathrm{s}}=0\right)$, which is produced by elastic scattering off non-magnetic impurities:

$$
\rho_{\mathrm{e}}=\frac{1}{\sigma_{\mathrm{e}}}, \quad \sigma_{\mathrm{e}}=2 e^{2} \nu D
$$

where $D=v_{\mathrm{F}}^{2} \tau_{\mathrm{e}} / 3$ is the diffusion coefficient. The second term is the contribution to the resistivity due to the scattering off magnetic impurities:

$$
\Delta \rho_{\mathrm{K}}=\frac{3 n_{\mathrm{s}}}{e^{2} \nu v_{\mathrm{F}}^{2}} \int \operatorname{Im} \overline{T(\varepsilon)} \frac{d \varepsilon}{4 T \cosh ^{2} \varepsilon / 2 T}
$$

The scattering T-matrix in Eq. (24) has different structure in the limits of high $\left(T \gg T_{\mathrm{K}}\right)$ and low $\left(T \ll T_{\mathrm{K}}\right)$ temperatures. We study these two limits below.

\section{A. High concentration of magnetic impurities, $T_{\mathrm{sg}} \gg T_{\mathrm{K}}$}

At high temperature $T \gg T_{\mathrm{K}}$ the scattering of electrons off magnetic impurities is described by the Born approximation with the exchange constant renormalized according to Eq. (2). In this case the $T$-matrix is (see Appendix [B]:

$$
\operatorname{Im} T(\varepsilon)=\pi \nu \mathcal{J}^{2} \int K(\omega) \frac{1+e^{\varepsilon / T}}{1+e^{(\varepsilon-\omega) / T}} \frac{d \omega}{2 \pi},
$$

where $K(\omega)$ is the Fourier transform of the spin correlation function, defined by Eq. (3). Substituting $\operatorname{Im} T(\varepsilon)$ into Eq. (24), we obtain the Kondo contribution to the Drude conductivity:

$$
\Delta \rho_{\mathrm{K}}=\frac{3 n_{\mathrm{s}} \mathcal{J}^{2}}{2 e^{2} v_{\mathrm{F}}^{2}} \int \overline{K(\omega)} \frac{\omega}{T} \frac{d \omega}{1-e^{-\omega / T}} .
$$

We emphasize that Eq. (26) is valid if the distance between impurities is much larger than the Fermi wavelength. At the same time, Eq. (26) describes the resistivity in metals with arbitrary structure and strength of interaction between magnetic impurities 22 We perform further calculations using the spin correlation function $\overline{K(\omega)}$ given by Eq. (15), which was derived within the virial expansion.

Using $\bar{K}(\omega)$, calculated within the virial expansion Eq. (15), and the Kondo renormalized exchange constant $\mathcal{J}$, see Eq. (2), we obtain

$$
\Delta \rho_{\mathrm{K}}=\frac{12 \pi n_{\mathrm{s}}}{e^{2} v_{\mathrm{F}}^{2} \nu^{2}} \frac{S(S+1)}{\ln ^{2}\left(T / T_{\mathrm{K}}\right)}\left(1-\alpha_{S} \frac{T_{\mathrm{sg}}}{T}\right) .
$$

Here numerical coefficient $\alpha_{S}$ is given by the following integral:

$$
\alpha_{S}=\int_{-\infty}^{+\infty}\left(1-\sum_{J J^{\prime}}^{2 S} \frac{y\left(\epsilon_{J}-\epsilon_{J^{\prime}}\right) e^{-y \epsilon_{J}}}{1-e^{-y\left(\epsilon_{J}-\epsilon_{J^{\prime}}\right)}} \frac{(2 J+1) A_{J J^{\prime}}}{S(S+1) Z(y)}\right) \frac{d y}{y^{2}}
$$

where $\epsilon_{J}, Z(y)$ and $A_{J J^{\prime}}$ are defined by Eqs. (11), (13a) and $13 \mathrm{~b}$ respectively. We emphasize that the integral in Eq. (28) converges near $y=0$. The values of $\alpha_{S}$ are presented in Table [

Equation (27) is similar to the results of Refs. 8, 17. Unlike Ref. 17, Eq. (27) takes into account the Kondo renormalization of the exchange constant, Eq. (2), and of the RKKY interaction, Eq. (10). The advantage of Eq. (27) in comparison with Ref. 8 is in a consistent definition of the energy scale $T_{\mathrm{sg}}$ and in an accurate procedure of the averaging over states of magnetic impurities, which allowed us to calculate the numerical factor $\alpha_{S}$.

In metals with low Kondo temperature, $T_{\mathrm{K}} \lesssim T_{\mathrm{sg}}$, the competition between the Kondo effect and the effect of RKKY interaction results in a maximum of the resistivity as a function of temperature. If $T_{\mathrm{K}} \ll T_{\mathrm{sg}}$, then the 
TABLE I: Values of the numerical coefficient $\alpha_{S}$, Eq. (28), for several values of $S$.

\begin{tabular}{c|c|c|c|c|c|c|c|}
\hline \hline$S$ & $1 / 2$ & 1 & $3 / 2$ & 2 & $5 / 2$ & 3 & $7 / 2$ \\
\hline$\alpha_{S}$ & 1.10 & 1.52 & 1.85 & 2.11 & 2.33 & 2.52 & 2.69 \\
\hline \hline
\end{tabular}

maximum occurs at

$$
T^{*} \simeq \frac{\alpha_{S}}{2} T_{\mathrm{sg}} \ln \frac{T_{\mathrm{sg}}}{T_{\mathrm{K}}}
$$

see also Ref. 78 9. We notice that temperature $T^{*}$ is within the region of applicability of the virial expansion, used in the derivation of Eq. (27).

As temperature $T$ approaches and crosses $T_{\mathrm{sg}}$, intrinsic random magnetic field develops, and the renormalization of the exchange constant is stopped at $\mathcal{J} \simeq$ $2 /\left(\nu \ln \left(T_{\mathrm{sg}} / T_{\mathrm{K}}\right)\right)$. Simultaneously, the virial expansion breaks down, and the collective modes of spin system have to be considered in the derivation of the temperature dependence of the resistivity.

At present, there is no theory of metallic spin glasses, which would provide us with $\overline{K(\omega)}$ in a broad range of $\omega$. Thus, the explicit form of $\Delta \rho_{\mathrm{K}}(T)$, see Eq. (26), is not known. We expect that $\Delta \rho_{\mathrm{K}}$ continues to decrease with temperature $T$ decreasing, as the dynamics of the local magnetic moments gets progressively suppressed at lower temperatures. In the mean field picture, each of the spins at $T=0$ is subject to a finite field, so that such dynamics is fully suppressed. In this case, the limiting value of $\Delta \rho_{\mathrm{K}}$ at $T=0$ can be estimated as

$$
\Delta \rho_{\mathrm{K}}(T=0)=\frac{12 \pi n_{\mathrm{s}}}{e^{2} v_{\mathrm{F}}^{2} \nu^{2}} \frac{S^{2}}{\ln ^{2}\left(T_{\mathrm{sg}} / T_{\mathrm{K}}\right)}
$$

(The quenching of the spin flips leads to the replacement of $S(S+1)$ factor, see Eq. (27), by the factor $S^{2}$ here.) The picture leading to Eq. (30) can not be valid for the "tightest" pairs of magnetic moments with the characteristic interaction energy significantly exceeding $T_{\mathrm{sg}}$. It is not clear to us at the moment even in which direction the estimate (27) changes due to the deviations from the mean field description.

\section{B. Low concentration of magnetic impurities, $T_{\mathrm{sg}} \ll T_{\mathrm{K}}$}

At high temperature $\left(T \gg T_{\mathrm{K}}\right)$, the resistivity is described by Eq. (27) even in the limit $T_{\mathrm{K}} \gg T_{\mathrm{sg}}$. Nevertheless, the effect of interaction between magnetic impurities is small due to the factor $T_{\mathrm{sg}} / T$ in Eq. (27), and the maximum of the resistivity does not occur at $T>T_{\mathrm{K}}$. We show that at temperatures $T \lesssim T_{\mathrm{K}}$ the resistivity also monotonically increases as temperature decreases.

For this purpose we use the following form of the imaginary part of the scattering $T$-matrix: ${ }^{23}$

$$
\operatorname{Im} T(\varepsilon)=\frac{1}{\pi \nu}-\operatorname{Im} \tilde{T}(\varepsilon)
$$

Here the first term is the contribution to the $T$-matrix in the unitary limit and $\tilde{T}(\varepsilon)$ is the $T$-matrix, written for the residual interaction between conduction electrons and magnetic impurities. The form of $\tilde{T}(\varepsilon)$ differs for spin impurity $S=1 / 2$ (exactly screened magnetic impurities) and for $S>1 / 2$ (underscreened magnetic impurities). ${ }^{23}$ We consider the two cases in more details below.

\section{Spin $S=1 / 2$ impurities}

In this case the majority of magnetic impurities are completely screened and the RKKY type interaction between them is absent (only a small part $\propto T_{\mathrm{sg}} / T_{\mathrm{K}}$ of magnetic impurities form coupled states with binding energy exceeding $T_{\mathrm{K}}$ ). Therefore the contribution to the resistivity is determined by the $T$-matrix of a single magnetic impurity, which is well studied at $T \ll T_{\mathrm{K}} 23.24$ We present the results for convenience. The scattering matrix for the residual interaction between conduction electrons and magnetic impurities has the form:

$$
\operatorname{Im} \tilde{T}(\varepsilon)=\frac{1}{\nu} \frac{9 \pi}{8 T_{\mathrm{K}}^{2}}\left(3 \varepsilon^{2}+\pi^{2} T^{2}\right) .
$$

Substituting Eqs. (31) and (32) into Eq. (24) and performing integration over energy $\varepsilon$, we obtain: ${ }^{23}$

$$
\Delta \rho_{\mathrm{K}}=\Delta \rho_{\mathrm{U}}\left[1-\frac{9 \pi^{4}}{4} \frac{T^{2}}{T_{\mathrm{K}}^{2}}\right]
$$

Here the factor

$$
\Delta \rho_{\mathrm{U}}=\frac{3}{\pi} \frac{n_{\mathrm{S}}}{e^{2} \nu^{2} v_{\mathrm{F}}^{2}}
$$

corresponds to the unitary contribution to the resistivity at $T=0$. According to Eq. (33) at finite temperature the resistivity contains corrections to Eq. (34), which are proportional to $T^{2} / T_{\mathrm{K}}^{2}$.

We also notice that the coefficients in Eq. (33) contain small corrections $T_{\mathrm{sg}} / T_{\mathrm{K}}$ due to magnetic impurities, which form coupled states with binding energies exceeding the Kondo temperature. Two possibilities exist: i) if the coupled state is a singlet, these impurities do not affect electron transport; ii) if the coupled state is a triplet, it becomes screened and again leads to a $T^{2}$ dependence of the resistivity on temperature ${ }^{25}$ We emphasize that the ratio of the number of such impurities to the total number of magnetic impurities is small as $T_{\mathrm{sg}} / T_{\mathrm{K}}$.

As the impurity concentration $n_{\mathrm{s}}$ increases, and consequently $T_{\mathrm{sg}}$ increases, the system of magnetic impurities with spin $S=1 / 2$ undergoes a quantum phase transi$\operatorname{tion}^{26}$ to a spin glass state.

\section{Impurities with $S>1 / 2$}

As was shown in ref. 24], at $T \ll T_{\mathrm{K}}$ the residual coupling of conduction electrons with magnetic impurities is 
described by the exchange Hamiltonian Eq. (10) with effective impurity spin, $\tilde{S}=S-1 / 2$, and the renormalized exchange constant

$$
\tilde{\mathcal{J}}=\frac{2}{\nu \ln T_{\mathrm{K}} / T} .
$$

This coupling results in the RKKY-like interaction between magnetic impurities, which may be written in the form of the Hamiltonian given by Eq. (7) with the effective spin $\tilde{S}$ and the strength $V(R)$ of the RKKY interaction defined as a solution of the following equation:

$$
V(R)=\frac{2}{\pi \nu R^{3}} \frac{1}{\ln ^{2}\left[T_{\mathrm{K}} / V(R)\right]} .
$$

Using Eq. (36) we estimate the typical value of the interaction between magnetic impurities. Within logarithmic accuracy we have

$$
\tilde{T}_{\mathrm{sg}} \simeq \frac{2 n_{\mathrm{s}}}{\pi \nu \ln ^{2}\left(T_{\mathrm{K}} \nu / n_{\mathrm{s}}\right)},
$$

cf. Eq. (10).

The imaginary part of the $\tilde{T}(\varepsilon)$-matrix is given by Eq. (25) with the impurity spin $\tilde{S}=S-1 / 2$, exchange constant $\mathcal{J}$ given by Eq. (35), and the typical value of the RKKY interaction between impurities $\tilde{T}_{\mathrm{sg}}$ given by Eq. (37). Substituting $\operatorname{Im} \tilde{T}(\varepsilon)$ from Eq. (25) with the modified parameters into Eq. (31) and using Eqs. (26) and (34), we obtain

$$
\Delta \rho_{\mathrm{K}}=\Delta \rho_{\mathrm{U}}\left[1-4 \pi^{2} \frac{S^{2}-1 / 4}{\ln ^{2} T_{\mathrm{K}} / T}\left(1-\alpha_{S-1 / 2} \frac{\tilde{T}_{\mathrm{Sg}}}{T}\right)\right] .
$$

Unlike the previously analyzed case of high impurity concentration, $T_{\mathrm{sg}} \gg T_{\mathrm{K}}$, here the temperature dependence of the resistivity remains monotonic. The leading contribution to the (negative) derivative $d \rho / d T$ in the temperature interval $\tilde{T}^{*} \lesssim T \lesssim T_{\mathrm{K}}$ comes from the Kondo renormalization of the exchange constant; here the characteristic temperature $\tilde{T}^{*}$ is

$$
\tilde{T}^{*} \simeq \frac{\alpha_{S-1}}{2} \tilde{T}_{\mathrm{sg}} \ln \frac{T_{\mathrm{K}}}{\tilde{T}_{\mathrm{sg}}} .
$$

Below $T^{*}$, the derivative $d \rho / d T$ is determined by the interaction between magnetic impurities.

As temperature approaches $\tilde{T}_{\mathrm{sg}}$, the virial expansion ceases to be valid, and the system may attain a spin glass state. Unlike the case of higher impurity concentration $\left(T_{\mathrm{sg}} \gg T_{\mathrm{K}}\right)$, here we expect the dependence $\rho(T)$ to level off at $T \lesssim T_{\mathrm{sg}}$. This difference stems from the behavior of the scattering matrix, see Eq. (31), in the vicinity of the unitary limit. If each of the local moments is quenched individually, then the saturation would occur at

$$
\Delta \rho_{\mathrm{K}}=\Delta \rho_{\mathrm{U}}\left[1-4 \pi^{2} \frac{(S-1 / 2)^{2}}{\ln ^{2} T_{\mathrm{K}} / T_{\mathrm{sg}}}\right] .
$$

The collective modes existing in the spin glass state would result, however, in deviations from Eq. (40).

To summarize Section III the Kondo contribution to the Drude resistivity is a non-monotonic function of temperature only in the case of relatively high concentration of the magnetic impurities, i.e., at $T_{\mathrm{sg}} \gtrsim T_{\mathrm{K}}$. In the opposite case, this contribution increases monotonically with the decrease of temperature, at any value of the spin $S$ of magnetic impurities.

\section{PHASE RELAXATION RATE}

Weak magnetic fields suppress the interference contribution to the conductivity, the difference

$$
\Delta \sigma_{\mathrm{wl}}=\sigma(B=0)-\sigma\left(B \gg B_{\mathrm{o}}\right)
$$

in the conductivity at zero magnetic field $B=0$ and at sufficiently strong magnetic field $B \gg B_{\mathrm{o}}$ is called the weak localization correction to the conductivity. The characteristic value $B_{\circ}$ of magnetic field, which suppresses the weak localization is

$$
B_{\mathrm{o}}=\frac{\hbar c}{e} \sqrt{\frac{1}{D \tau_{\mathrm{s}} A}},
$$

where $A$ is the cross-section area of the wire. Throughout this Section, we assume that the scattering off magnetic impurities dominates over all other mechanisms of the electron phase relaxation.

We calculate $\Delta \sigma_{\mathrm{wl}}$ of a metal with magnetic impurities and strong spin orbit coupling. In this case only the singlet component $\mathcal{C}_{s}$ of the Cooperon remains finite, 18 all other Cooperon components are suppressed by the spinorbit interaction. The weak (anti)localization correction to the conductivity is given by:27,28

$$
\Delta \sigma_{\mathrm{wl}}=\frac{e^{2} D}{\hbar} \int \frac{d \varepsilon d \varepsilon_{1} d \varepsilon_{2}}{(2 \pi)^{3}} \frac{d^{d} \boldsymbol{q}}{(2 \pi)^{d}} \frac{\mathcal{C}_{s}^{+}\left(\begin{array}{l}
\varepsilon, \varepsilon_{1} \\
\varepsilon_{2}, \varepsilon
\end{array} ;\right)}{2 T \cosh ^{2} \varepsilon / 2 T} .
$$

Neglecting the RKKY interaction between magnetic impurities, we have the following expression for the Cooperon:

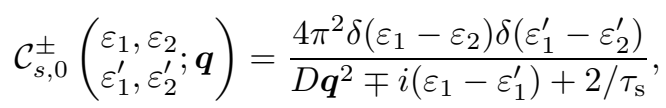

where $1 / \tau_{\mathrm{s}}$ is the electron scattering rate off magnetic impurities and is defined by Eq. (6) 18 In calculating the WL correction, we assume that the rate $1 / \tau_{\mathrm{s}}$ is higher than the Korringa relaxation rate of the magnetic impurities, see Ref. 13 for further discussion. Performing integration over the momentum $\boldsymbol{q}$ in a one dimensional case, $d=1$ in Eq. (43), which adequately describes wires of the cross-sectional area $A \lesssim D \tau_{\mathrm{s}}$, we obtain

$$
\Delta \sigma_{\mathrm{wl}}^{(0)}=\frac{e^{2}}{2 \pi \hbar} \sqrt{\frac{D \tau_{\mathrm{s}}}{2}} .
$$


We study the effect of interaction between spins of magnetic impurities on the weak localization correction to the conductivity. As we have already mentioned, the interaction between magnetic impurities lifts the degeneracy of impurity spin states, and therefore it is reminiscent to the Zeeman effect of external magnetic field. The Zeeman splitting of spin states of magnetic impurities affects the WL correction to the conductivity, 12.13 if the splitting is larger than either temperature $T$ or phase relaxation rate $1 / \tau_{\mathrm{s}}$. Similarly, the RKKY interaction between two impurities starts to affect the WL correction if the RKKY interaction strength exceeds $T$ or $1 / \tau_{\mathrm{s}}$. We calculate the WL correction at temperatures $T$ higher than $T_{\mathrm{sg}}$ ( or $\tilde{T}_{\mathrm{sg}}$ ), so that only a small number of magnetic impurity pairs satisfy this condition, and therefore the virial expansion is applicable.

We notice that because of the RKKY interaction between impurity spins, the scattering processes may change electron energy and, particularly, may switch the position of the Cooperon poles in energy plane with respect to the real axis. These processes result in mixing of the Cooperon components $\mathcal{C}^{+}$and $\mathcal{C}^{-}$which have different analyticity, see e.g. Eq. (44). The full equation for the Cooperon is

$$
\begin{array}{r}
\hat{\mathcal{C}}_{s}\left(\begin{array}{c}
\varepsilon_{1}, \varepsilon_{2} \\
\left.\varepsilon_{1}^{\prime}, \varepsilon_{2}^{\prime} ; \boldsymbol{q}\right)
\end{array}\right) \hat{\mathcal{C}}_{s, 0}\left(\begin{array}{l}
\varepsilon_{1}, \varepsilon_{2} \\
\varepsilon_{1}^{\prime}, \varepsilon_{2}^{\prime}
\end{array}\right)+\int \frac{d \varepsilon_{3} d \varepsilon_{3}^{\prime} d \varepsilon_{4} d \varepsilon_{4}^{\prime}}{(2 \pi)^{4}} \\
\times \hat{\mathcal{C}}_{s, 0}\left(\begin{array}{c}
\varepsilon_{1}, \varepsilon_{3} \\
\varepsilon_{1}^{\prime}, \varepsilon_{3}^{\prime}, \boldsymbol{q}
\end{array}\right) \hat{\Sigma}\left(\begin{array}{l}
\varepsilon_{3}, \varepsilon_{4} \\
\varepsilon_{3}^{\prime}, \varepsilon_{4}^{\prime}
\end{array}\right) \hat{\mathcal{C}}_{s}\left(\begin{array}{c}
\varepsilon_{4}, \varepsilon_{2} \\
\varepsilon_{4}^{\prime}, \varepsilon_{2}^{\prime}, \boldsymbol{q}
\end{array}\right) .
\end{array}
$$

The diagonal elements of the $2 \times 2$ matrix $\hat{\mathcal{C}}_{s}$ are $\mathcal{C}_{s}^{ \pm}$. The matrix

$$
\hat{\mathcal{C}}_{s, 0}(\cdot, \boldsymbol{q})=\left(\begin{array}{cc}
\hat{\mathcal{C}}_{s, 0}^{+}(\cdot, \boldsymbol{q}) & 0 \\
0 & \hat{\mathcal{C}}_{s, 0}^{-}(\cdot, \boldsymbol{q})
\end{array}\right)
$$

is the Cooperon to the zeroth order in the RKKY interaction, see Eq. (44), while the self energy

$$
\hat{\Sigma}_{s}(\cdot)=\left(\begin{array}{cc}
\Sigma_{s}^{\mathrm{rr}}(\cdot) & \Sigma_{s}^{\mathrm{ra}}(\cdot) \\
\Sigma_{s}^{\mathrm{ar}}(\cdot) & \Sigma_{s}^{\mathrm{aa}}(\cdot)
\end{array}\right)
$$

contains the higher-order RKKY contributions.

To evaluate the first term of the virial expansion, we may account for $\hat{\Sigma}_{s}$ by the first-order iteration of the solution of Eq. (46). The self energy $\hat{\Sigma}$ must be calculated up to the first order in the RKKY interaction. In fact, it is sufficient to evaluate the upper diagonal element $\Sigma_{s}^{\mathrm{rr}}(\cdot)$ of the matrix $\hat{\Sigma}_{s}(\cdot)$ and write the Cooperon as:

$$
\begin{aligned}
\mathcal{C}_{s}^{+}\left(\begin{array}{l}
\varepsilon, \varepsilon_{1} \\
\varepsilon_{2}, \varepsilon
\end{array} ; \boldsymbol{q}\right) & =\frac{1}{D \boldsymbol{q}^{2}+2 / \tau_{\mathrm{s}}}+\frac{1}{D \boldsymbol{q}^{2}+2 / \tau_{\mathrm{s}}-i\left(\varepsilon-\varepsilon_{2}\right)} \\
& \times \Sigma_{s}^{\mathrm{rr}}\left(\begin{array}{l}
\varepsilon, \varepsilon_{1} \\
\varepsilon_{2}, \varepsilon
\end{array}\right) \frac{1}{D \boldsymbol{q}^{2}+2 / \tau_{\mathrm{s}}-i\left(\varepsilon_{1}-\varepsilon\right)},
\end{aligned}
$$

with (see Appendix C)

$$
\begin{aligned}
& \Sigma_{s}^{\mathrm{rr}}\left(\begin{array}{cc}
\varepsilon, & \varepsilon_{1} \\
\varepsilon_{2}, & \varepsilon
\end{array}\right)=-4 \pi^{2} \nu n_{\mathrm{s}} \mathcal{J}^{2} \int d \omega \frac{\left(e^{\varepsilon / T}+1\right) \delta \overline{K_{2}(\omega)}}{e^{(\varepsilon-\omega) / T}+1} \\
& \quad \times\left\{\delta\left(\varepsilon_{1}+\omega-\varepsilon\right) \delta\left(\varepsilon-\varepsilon_{2}-\omega\right)+\delta\left(\varepsilon_{1}-\varepsilon\right) \delta\left(\varepsilon-\varepsilon_{2}\right)\right\} .
\end{aligned}
$$

Then, the weak localization correction to the conductivity is obtained by substituting this expression for $\mathcal{C}_{s}^{+}(\cdot ; \boldsymbol{q})$ into Eq. (43) and performing integration over momentum $\boldsymbol{q}$ and energies. As we discussed in the previous Section, details of the structure of the spin correlation function $\delta \overline{K_{2}(\omega)}$ in Eq. (50) depend on the relation between temperature $T$, Kondo temperature $T_{\mathrm{K}}$ and the typical energy of interaction between impurities $T_{\mathrm{sg}}$. Some of these limits are discussed below.

\section{A. High concentration of magnetic impurities, $T_{\mathrm{sg}} \gg T_{\mathrm{K}}$}

At temperatures $T \gg T_{\mathrm{K}}$ the scattering of electrons off magnetic impurities is described by the Born approximation with the renormalized exchange constant, Eq. (2). We substitute $\delta \overline{K_{2}(\omega)}$ (the first order term in the RKKY interaction) from Eq. (16) into Eq. (50), and using Eqs. (43) and (49), we obtain the weak localization correction to the conductivity [see Appendix D] for more details]. We distinguish three temperature domains for the WL correction to the conductivity $\Delta \sigma_{\mathrm{wl}}$.

In the highest of the three domains, $T \gg 2 / \tau_{\mathrm{s}}$, the weak localization correction has the form

$$
\Delta \sigma_{\mathrm{wl}}=\frac{e^{2}}{2 \pi \hbar} \sqrt{\frac{D \tau_{\mathrm{s}}}{2}}\left(1+\frac{\pi(4 S+1)(4 S+3)}{120(2 S+1)} T_{\mathrm{sg}} \tau_{\mathrm{s}}\right)
$$

with the second term in the parentheses coming from the RKKY interaction. The use of Eq. (10) for $T_{\mathrm{sg}}$ and of the estimate for the Kondo-renormalized electron spin relaxation rate,

$$
\frac{1}{\tau_{\mathrm{s}}}=\frac{8 \pi n_{\mathrm{s}}}{\nu} \frac{S(S+1)}{\ln ^{2} T / T_{\mathrm{K}}}
$$

allows us to estimate $T_{\mathrm{sg}} \tau_{\mathrm{s}}$ as

$$
T_{\mathrm{sg}} \tau_{\mathrm{s}}=\frac{1}{4 \pi^{2} S(S+1)} \frac{\ln ^{2}\left(T / T_{\mathrm{K}}\right)}{\ln ^{2}\left(v_{\mathrm{F}} n_{\mathrm{s}}^{1 / 3} / T_{\mathrm{K}}\right)}
$$

We see now that the correction due to the RKKY interaction only weakly depends on temperature, and is numerically small.

It is curious to notice that the second term in Eq. (51) is almost independent of $n_{\mathrm{s}}$. This term takes into account the fact that the contribution to the phase relaxation rate is suppressed, if a scattering process results in energy exchange larger than $1 / \tau_{\mathrm{s}}$. The reduction of the phase relaxation rate leads to the enhancement of the weak localization correction to the conductivity, as shown in Eq. (51). The number of impurities with the splitting of energy states larger than $1 / \tau_{\mathrm{s}}$ constitute only $T_{\mathrm{sg}} \tau_{\mathrm{s}}$ part of the total number of magnetic impurities. We emphasize that the accidental numerical smallness of the RKKY-induced correction $\propto T_{\mathrm{sg}} \tau_{\mathrm{s}}$ justifies the use of the conventional theory ${ }^{18}$ of the weak localization in the 
presence of magnetic impurities in the considered temperature domain.

In the second temperature domain $T_{\mathrm{sg}} \lesssim T \lesssim 1 / \tau_{\mathrm{s}}$, the WL correction equals

$$
\begin{aligned}
\Delta \sigma_{\mathrm{wl}} & =\frac{e^{2}}{2 \pi \hbar} \sqrt{\frac{D \tau_{\mathrm{s}}}{2}}\left(1+\frac{\alpha_{S}}{2} \frac{T_{\mathrm{sg}}}{T}\right) \\
& \propto \ln \frac{T}{T_{\mathrm{K}}}\left(1+\frac{\alpha_{S}}{2} \frac{T_{\mathrm{sg}}}{T}\right) .
\end{aligned}
$$

Here the numerical factor $\alpha_{S}$ is defined in Eq. (28), and $T_{\mathrm{sg}}$ is given by Eqs. (10). The result shown in Eq. (54) has a similar structure to the expression for the resistivity correction Eq. (27). The dependence $\Delta \sigma_{\mathrm{wl}}$ vs. $T$ has a minimum at temperature $T^{*}$ defined in Eq. (29). This minimum results from the competition between two opposite trends: with the reduction of temperature, $\tau_{\mathrm{s}}$ gets shorter, see Eq. (52), while the stronger-bound impurity pairs stop affecting $\Delta \sigma_{\mathrm{wl}}$. Note that due to the relation between $T_{\mathrm{sg}}$ and $1 / \tau_{\mathrm{s}}$, see Eq. (53), the second temperature domain is rather wide.

The third temperature domain corresponds to the spin glass state of the magnetic impurities. With temperature decreasing to $T_{\mathrm{sg}}$, the virial correction becomes large and Eq. (54) is no longer applicable. At such temperature a spin glass transition is expected. Below the transition, $\Delta \sigma_{\mathrm{wl}}$ is still determined by the spin correlation function, Eq. (3). Similar to the discussion of the resistivity in Section IIII we expect a monotonic increase and saturation of the WL correction. The limiting value of $\Delta \sigma_{\mathrm{wl}}$ at $T=0$ was estimated in Ref. 29, where quenching of the dynamics of each of the local moments was assumed. Deviations from such a simple picture of the spin glass state would result in a different value of $\Delta \sigma_{\mathrm{wl}}(T=0)$.

\section{B. Low concentration of magnetic impurities, $T_{\mathrm{sg}} \ll T_{\mathrm{K}}$}

At $T \gg T_{\mathrm{K}}$, the weak localization correction to the conductivity is still given by Eq. (54). However, now the effect of the RKKY interaction on $\Delta \sigma_{\mathrm{wl}}$ is small, and the WL correction to the conductivity decreases monotonically with the decrease of temperature.

At temperature $T \ll T_{\mathrm{K}}$ the scattering off a single impurity approaches the unitary limit. The potential scattering characterizing the unitary limit, does not destroy phase coherence and thus does not affect the WL correction. Therefore, at $T \ll T_{\mathrm{K}}$ only small deviations from the unitary limit determine $\Delta \sigma_{\mathrm{wl}}$. In this section we show that $\Delta \sigma_{\mathrm{wl}}$ increases monotonically as temperature decreases in the domain $T \ll T_{\mathrm{K}}$; the details of the temperature dependence are different for $S=1 / 2$ and $S>1 / 2$.

Comparing the behavior of WL correction in the domains of low and high temperatures, we conclude that the correction must have a minimum at $T \sim T_{\mathrm{K}}$, assuming that the scattering off magnetic impurities dominates the electron phase relaxation.

\section{Spin $S=1 / 2$}

At $T=0$, the spins of magnetic impurities are completely screened and do not contribute to the phase relaxation of the conduction electrons. At finite but small temperatures, $T \ll T_{\mathrm{K}}$, the residual local electronelectron interaction facilitated by local moments leads to the electron relaxation which affects the Cooperon pole:

$$
\mathcal{C}_{s, 1 / 2}^{+}\left(\begin{array}{l}
\varepsilon, \varepsilon_{1} \\
\varepsilon_{2}, \varepsilon
\end{array} ;\right)=\frac{4 \pi^{2} \delta\left(\varepsilon-\varepsilon_{1}\right) \delta\left(\varepsilon-\varepsilon_{2}\right)}{D \boldsymbol{q}^{2}-i\left(\varepsilon_{1}-\varepsilon\right)+\tilde{\Gamma}(\varepsilon)}
$$

Here the relaxation rate

$$
\tilde{\Gamma}(\varepsilon)=\frac{9 \pi}{8} \frac{n_{\mathrm{s}}}{\nu} \frac{3 \varepsilon^{2}+\pi^{2} T^{2}}{T_{\mathrm{K}}^{2}} .
$$

Because the interaction responsible for the relaxation is local, the typical energy transferred in a scattering event is $\Delta \varepsilon \sim T$, and therefore $\tilde{\Gamma}(\varepsilon) / \Delta \varepsilon \ll T T_{\mathrm{sg}} / T_{\mathrm{K}}^{2} \ll 1$. Under these conditions, the Cooperon relaxation rate $\tilde{\Gamma}(\varepsilon)$ is just twice the one-electron relaxation rate.

Substituting $\mathcal{C}^{+}(\cdot, q)$ from Eq. (55) into Eq. (43), we obtain

$$
\Delta \sigma_{\mathrm{wl}}=\frac{e^{2}}{2 \pi \hbar} \int \sqrt{\frac{D}{\tilde{\Gamma}(\varepsilon)}} \frac{d \varepsilon}{4 T \cosh ^{2} \varepsilon / 2 T} .
$$

According to Eq. (57), in the absence of other phase relaxation mechanisms, the weak localization correction would vary as $1 / T$ at $T \lesssim T_{\mathrm{K}}$ :

$$
\Delta \sigma_{\mathrm{wl}} \approx 0.022 \frac{e^{2}}{\hbar} \sqrt{\frac{\nu D}{n_{\mathrm{s}}}} \frac{T_{\mathrm{K}}}{T} .
$$

We notice, that similar to Eq. (33), there are small corrections of the order of $T_{\mathrm{sg}} / T_{\mathrm{K}}$ to the numerical coefficient in Eq. (58). The corrections originate from the rare configurations of "tight" pairs of magnetic impurities, which form singlet or triplet states with binding energy exceeding the Kondo temperature.

\section{2. $\operatorname{Spin} S>1 / 2$}

The residual coupling between conduction electrons and magnetic impurities with $S>1 / 2$ is still described by the exchange Hamiltonian Eq. (1) with the reduced spin operator $\widetilde{S}=S-1 / 2$ and the renormalized exchange constant $\tilde{\mathcal{J}}$, see Eq. (35). In this case the electron scattering rate is

$$
\frac{1}{\tilde{\tau}_{\mathrm{s}}}=\frac{8 \pi n_{\mathrm{s}}}{\nu} \frac{S^{2}-1 / 4}{\ln ^{2} T_{\mathrm{K}} / T} .
$$

The coupling $\tilde{\mathcal{J}}$ also results in the RKKY interaction between the partially screened local moments, which is 
represented by the Hamiltonian Eq. (7) with $\hat{\mathbf{S}}$ replaced by $\hat{\tilde{\mathbf{S}}}$. The strength of the RKKY interaction is determined by the self-consistent equation (36).

To calculate the weak localization correction to the conductivity, we use Eqs. (43) and (49) with the self energy $\Sigma_{\mathrm{rr}}$ in the form of Eq. (50). The spin correlation function $\delta \overline{K_{2}(\omega)}$ in Eq. (50) describes correlations of $\tilde{S}$ spins with the appropriately replaced exchange constant Eq. (35) and the typical value $\tilde{T}_{\text {sg }}$ of the RKKY potential, Eq. (37).

Similar to Section ஹA we can define three domains for the temperature dependence of the weak localization correction. At high temperature $T \gtrsim 2 / \tilde{\tau}_{\mathrm{s}}$, we obtain

$$
\Delta \sigma_{\mathrm{wl}}=\frac{e^{2}}{2 \pi \hbar} \sqrt{\frac{D \tilde{\tau}_{\mathrm{s}}}{2}}\left[1+\frac{\pi\left(16 S^{2}-1\right)}{240 S} \tilde{T}_{\mathrm{sg}} \tilde{\tau}_{\mathrm{s}}\right] .
$$

Equation (60) is a counterpart of Eq. (51).

At lower temperatures, $\tilde{T}_{\mathrm{sg}} \lesssim T \lesssim 1 / \tilde{\tau}_{\mathrm{s}}$, we obtain [compare to Eq. (54)]:

$$
\Delta \sigma_{\mathrm{wl}}=\frac{e^{2}}{2 \pi \hbar} \sqrt{\frac{D \tilde{\tau}_{\mathrm{s}}}{2}}\left[1+\frac{\alpha_{S-1 / 2}}{2} \frac{\tilde{T}_{\mathrm{sg}}}{T}\right] .
$$

We notice that at $T \ll T_{\mathrm{K}}$ as temperature decreases the weak localization correction to the conductivity increases. We conclude that both the Kondo effect and the effect of interaction between magnetic impurities reduce the phase relaxation rate as temperature decreases. At $\tilde{T}^{*} \lesssim T \lesssim T_{\mathrm{K}}$ the temperature dependence of the weak localization correction to the conductivity is mainly determined by the Kondo effect, and at lower temperatures, $T \lesssim \tilde{T}^{*}$ it is determined by the interaction between magnetic impurities. At temperature below $\tilde{T}_{\text {sg }}$ a spin glass state may appear. Similar to the behavior of $\Delta \rho_{\mathrm{K}}(T)$, the weak localization correction increases monotonically with the decrease of temperature, and should saturate at $T \rightarrow 0$.

To summarize Section IV the weak localization correction to the conductivity is a non-monotonic function of temperature. At relatively high concentration of the magnetic impurities, i.e. at $T_{\mathrm{sg}} \gtrsim T_{\mathrm{K}}$, the positions of minimum in $\Delta \sigma_{\mathrm{wl}}$ and maximum in $\Delta \rho_{\mathrm{K}}$ roughly coincide, see Eqs. (27) and (54). In the opposite case, the minimum in $\Delta \sigma_{\mathrm{wl}}$ occurs at $T \simeq T_{\mathrm{K}}$.

\section{ENERGY RELAXATION RATE}

Free magnetic impurities are an intermediary for electron-electron scattering with small energy transfer ${ }^{3}$ We show that the RKKY interaction between magnetic impurities leads to the electron energy relaxation as a result of a single electron scattering off a magnetic impurity. Indeed, if the impurity interacts with one or more of its neighbors, a scattering process is accompanied by the energy exchange between conduction electrons and magnetic impurities.
In this Section we apply the virial expansion method to derive kinetic equations for the non-equilibrium distribution function of electrons in a dilute magnetic alloy. The virial expansion is justified for processes with large compared to $T_{\mathrm{sg}}$ energy transfer from an electron to the system of localized moments; here $T_{\mathrm{sg}}$ is the typical energy of interaction between magnetic impurities, see Eq. (10). We assume that the spin-orbit interaction is strong, in which case the electron distribution is independent of spin orientation, $f_{\uparrow}\left(t, \boldsymbol{r}, \varepsilon_{k}\right)=f_{\downarrow}\left(t, \boldsymbol{r}, \varepsilon_{k}\right) \equiv f\left(t, \boldsymbol{r}, \varepsilon_{k}\right)$.

First, we consider electron scattering by magnetic impurities belonging to a small-size pair. If the electron distribution function does not significantly vary on the length scale of the order of the pair size, then the corresponding scattering rate can be expressed in terms of the electron distribution function $f\left(t, r, \varepsilon_{k}\right)$ at the position $\mathbf{r}$ of the pair,

$$
\begin{aligned}
\Upsilon_{k k^{\prime}}^{J J^{\prime}}(t, \boldsymbol{r}) & =2 \pi \mathcal{J}^{2}(2 J+1) A_{J J^{\prime}} \delta\left(\varepsilon_{k}-\varepsilon_{k^{\prime}}+E_{J}-E_{J^{\prime}}\right) \\
& \times P_{J}(t, \boldsymbol{r}, V) f\left(t, \boldsymbol{r}, \varepsilon_{k}\right)\left(1-f\left(t, \boldsymbol{r}, \varepsilon_{k^{\prime}}\right)\right) .(62)
\end{aligned}
$$

Here $P_{J}(t, \boldsymbol{r}, V)$ is the distribution function for two magnetic impurities over quantum states characterized by the total spin $J$ of the pair, $E_{J}=V \epsilon_{J}$, see Eq. (11), and $A_{J J^{\prime}}$ is defined by Eq. (13b).

Having the rate $\Upsilon_{k k^{\prime}}^{J J^{\prime}}(\boldsymbol{r})$, we can write the kinetic equations for the distribution function of the pairs $P_{J}(V)$. Performing summation over all initial $(k, \alpha)$ and final $\left(k^{\prime}, \alpha^{\prime}\right)$ states of a scattered electron as well as over the final states of the pair, we obtain the following equation:

$$
\frac{d P_{J}(t, V)}{d t}=-\frac{4 \nu^{2}}{2 J+1} \sum_{J^{\prime}} \int d \varepsilon_{k} d \varepsilon_{k^{\prime}}\left(\Upsilon_{k k^{\prime}}^{J J^{\prime}}-\Upsilon_{k^{\prime} k}^{J^{\prime} J}\right)
$$

(we omit the position $\boldsymbol{r}$ of the pair in the argument of $P$ ). The normalization condition for $P_{J}(t, V)$ has the form

$$
\sum_{J=0}^{2 S}(2 J+1) P_{J}(t, V)=1 .
$$

In the stationary state, the distribution $P_{J}(t, V) \equiv$ $P_{J}(V)$ satisfies the equations

$$
P_{J+1}(V)=P_{J}(V) \frac{\int f(\varepsilon)\left(1-f\left(\varepsilon+V \epsilon_{J, J+1}\right)\right) d \varepsilon}{\int f\left(\varepsilon+V \epsilon_{J, J+1}\right)(1-f(\varepsilon)) d \varepsilon},
$$

where we use the shorthand notation $\epsilon_{J, J^{\prime}}=\epsilon_{J}-\epsilon_{J^{\prime}}$, and $\epsilon_{J}$ is defined by Eq. (11). If the system of magnetic impurities and electrons are at equilibrium with temperature $T$, the solution of Eq. [65) is the Gibbs distribution:

$$
P_{J}(V)=\frac{\exp \left(-V \epsilon_{J} / T\right)}{\sum_{J}(2 J+1) \exp \left(-V \epsilon_{J} / T\right)} .
$$

Next, we write the kinetic equation for the electron distribution function

$$
\left[\frac{\partial}{\partial t}-D \frac{\partial^{2}}{\partial^{2} \boldsymbol{r}}\right] f(t, \boldsymbol{r}, \varepsilon)=-\mathcal{I}\left(t, \varepsilon_{k}, V\right)
$$


where the electron collision integral has the form:

$$
\mathcal{I}\left(t, \varepsilon_{k}, V\right)=\nu \sum_{J J^{\prime}} \int\left(\Upsilon_{k k^{\prime}}^{J J^{\prime}}-\Upsilon_{k^{\prime} k}^{J^{\prime} J}\right) d \varepsilon_{k^{\prime}}
$$

with $\Upsilon_{k k^{\prime}}^{J J^{\prime}}$ given by Eq. (62).

We assume that the electron distribution function changes slowly with the coordinate $\boldsymbol{r}$, so that the collision integral $\mathcal{I}$ may be averaged over a small volume of the metal, where $f\left(t, r, \varepsilon_{k}\right)$ does not change much, but which contains many magnetic impurities. In this case we can perform averaging of the collision integral over the RKKY potential according to:

$$
\langle\mathcal{I}(t, \varepsilon)\rangle=\frac{4 T_{\mathrm{sg}}}{3} \int \frac{d V}{V^{2}} \mathcal{I}(t, \varepsilon, V)
$$

Substituting Eq. (62) into Eq. (68) and performing averaging according to Eq. (69), we obtain

$$
\langle\mathcal{I}(t, \varepsilon)\rangle=\frac{4 T_{\mathrm{sg}}}{3 \tau_{\mathrm{s}}} \sum_{J \neq J^{\prime}} \frac{2 J+1}{S(S+1)} A_{J J^{\prime}}\left|\epsilon_{J J^{\prime}}\right| \int \frac{d E}{E^{2}}\left\{f_{\varepsilon}\left(1-f_{\varepsilon-E}\right) P_{J}\left(t, \frac{-E}{\epsilon_{J J^{\prime}}}\right)-\left(1-f_{\varepsilon}\right) f_{\varepsilon-E} P_{J^{\prime}}\left(t, \frac{-E}{\epsilon_{J J^{\prime}}}\right)\right\}
$$

where we use notations $\epsilon_{J J^{\prime}}=\epsilon_{J}-\epsilon_{J^{\prime}}$ and $f_{\varepsilon}=f(t, \boldsymbol{r}, \varepsilon)$. In the derivation of the collision integral, we tacitly assumed that the transferred energy $E$ exceeds the width of spin states, given by the Korringa relaxation rate, $\hbar / \tau_{T} \propto T \nu^{2} \mathcal{J}^{2}(T)$.

The system of equations (63) and (67), with the scattering rates given by Eq. (62), the electron collision integral replaced by its average, Eq. (70), and with the initial conditions for $f(t=0, \varepsilon, \boldsymbol{r}), P_{J}(t=0, V)$ and the boundary conditions for $f(t, \varepsilon, \boldsymbol{r})$, define completely the kinetics of electrons and spins. In the stationary case $(\partial f / \partial t=0)$, one may use Eq. (65) instead of Eqs. (62) and (63). Note that the impurity average collision integral, Eq. (70), differs from the conventional collision integral for the electron-electron scattering. The $1 / E^{2}$ behavior of the kernel in Eq. (70) does not imply the scaling of the distribution function found in Ref. 2 .

The collision integral, Eq. (70), may be simplified for the electron energies $\varepsilon \gg T$. As a result, we obtain the following kinetic equation for the distribution of "hot" $(\varepsilon \gg T)$ electrons:

$$
D \frac{\partial^{2} f(\varepsilon, x)}{\partial x^{2}}=\frac{1}{\tau_{\mathrm{s}}} \int \chi(E)\{f(\varepsilon, x)-f(\varepsilon+E, x)\} \frac{d E}{T_{\mathrm{sg}}} .
$$

This equation is a version of a full kinetic equation, defined by Eqs. (63)- (70), which may be used for analysis of the high energy tail of the electron distribution function in a metal with magnetic impurities. The kernel $\chi(E)$ in Eq. (71) is asymmetric with respect to the energy transfer $E$ and for $|E| \gg T_{\mathrm{sg}}$ has the following form

$$
\chi(E)=\frac{16}{3} \frac{T_{\mathrm{sg}}^{2}}{E^{2}} \frac{e^{E / T}\left(e^{E / T}+1\right)}{\left(3 e^{E / T}+1\right)\left(3+e^{E / T}\right)} .
$$

According to Eq. (72) the probability for an electron to scatter with energy gain $(E<0)$ is exponentially small at $|E| \gg T$, while the rate of scattering with an energy loss $E \gg T$ scales as a power of transferred energy, $\propto T_{\mathrm{sg}}^{2} / E^{2}$.
We notice that because of the relatively slow decay of $\chi(E)$ with energy $E>0$, the relaxation of the number of non-equilibrium electrons occurs differently from the relaxation of their energy. We illustrate the energy transfer from electrons to the system of magnetic impurities by considering the following model problem. Assume, that initially the system of electrons and magnetic impurities is in equilibrium at temperature $T$, and then instantaneously the electron subsystem is brought out of equilibrium, so that the new distribution function is characterized by small deviation $\delta f(\varepsilon)$ from the equilibrium. The excess electron energy $W$ per unit volume may be defined as $W=2 \nu \int \varepsilon \delta f(\varepsilon) d \varepsilon$. The energy $W$ will decrease in time as the result of the energy redistribution between electron and impurity subsystems. Eventually, a new equilibrium with new temperature will establish. We calculate the reduction of the electron energy at the initial moment. The result is

$$
\begin{aligned}
\frac{d W}{d t}= & \frac{16 T_{\mathrm{sg}}}{3 \tau_{\mathrm{s}}} \int \frac{d E}{E} \frac{e^{2 E / T}-1}{\left(3 e^{E / T}+1\right)\left(3+e^{E / T}\right)} \\
& \times \int \frac{\nu \delta f(\varepsilon) \sinh \varepsilon / T}{\cosh E / T+\cosh \varepsilon / T} d \varepsilon .
\end{aligned}
$$

If the distribution of electrons at the initial moment was peaked near energy $\varepsilon_{0} \gg T$, e.g. $\nu \delta f(\varepsilon)=\alpha \delta\left(\varepsilon-\varepsilon_{0}\right)$, then the estimate of the energy reduction rate is $d W / d t=$ $\alpha T_{\mathrm{sg}} / \tau_{\mathrm{s}} \ln \varepsilon_{0} / T$. The characteristic collision rate is $\sim$ $1 / \tau_{\mathrm{s}}$ and the typical energy transferred in a collision is $T_{\mathrm{sg}}$. However, the range of the transferred energies is broad enough to result in a logarithmic dependence on $\varepsilon_{0} / T$.

In conclusion we emphasize that Eq. (72) is derived within the virial expansion and is valid for large energy transfer. We expect that beyond the virial expansion the kernel $\chi(E)$ remains to be a function of $E / T_{\mathrm{sg}}$ and $T / T_{\mathrm{sg}}$ :

$$
\chi(E)=\mathcal{F}\left(\frac{E}{T_{\mathrm{sg}}}, \frac{T}{T_{\mathrm{sg}}}\right)
$$




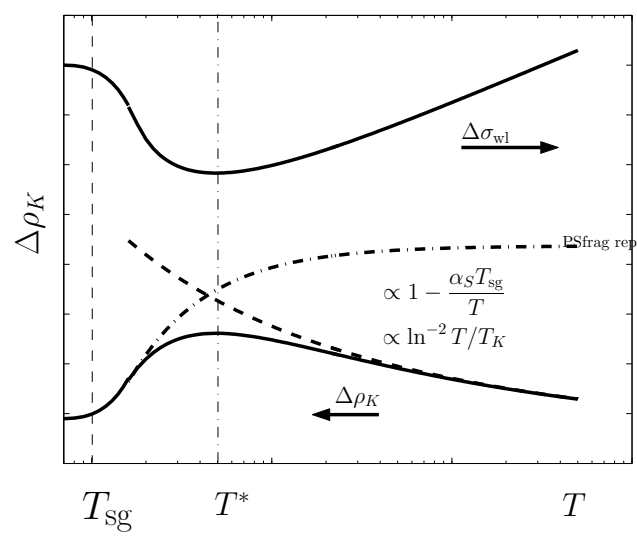

FIG. 1: Schematic picture of the Kondo contribution to the resistivity $\Delta \rho_{\mathrm{K}}$ and of the weak localization correction $\Delta \sigma_{\mathrm{wl}}$ to the conductivity in the limit $T_{\mathrm{sg}} \gg T_{\mathrm{K}}$. Both $\Delta \rho_{\mathrm{K}}$ and $\Delta \sigma_{\mathrm{wl}}$ are expected to saturate as $T$ approaches $T_{\mathrm{sg}}$.

Function $\mathcal{F}$ characterizes the excitation spectrum of a system of magnetic impurities at the energy scales relevant for the kinetics of conduction electrons. The study of its properties may provide important information about formation of spin glass states in metals with magnetic impurities.

\section{DISCUSSION AND CONCLUSIONS}

In this paper, we considered the effect of the RKKY interaction between Kondo impurities in a metal on kinetic properties of conduction electrons. Specifically, we evaluated the effect of interacting magnetic impurities on: the momentum relaxation rate and the corresponding contribution to the Drude resistivity of a metal $\Delta \rho_{K}$, the phase relaxation rate as defined by the weak localization correction $\Delta \sigma_{\mathrm{wl}}$ to the Drude conductivity, see Eq. (41), and the energy relaxation rate, which determines the relaxation of non-equilibrium electrons injected into a metal.

The overall temperature dependence of the momentum and phase relaxation rates differs for the cases of strong and weak RKKY interaction between the magnetic impurities.

If the interaction $T_{\mathrm{sg}}$ between the impurities separated by a typical distance $n_{\mathrm{s}}^{-1 / 3}$ is strong, $T_{\mathrm{sg}} \gg T_{\mathrm{K}}$, then the momentum and phase relaxation rates are nonmonotonic functions of temperature $T$, with the maxima at $T \simeq T^{*}$, see Eq. (29) [here $T_{\mathrm{K}}$ is the Kondo temperature for a single magnetic impurity, $n_{\mathrm{s}}$ is the concentration of these impurities, and energy $T_{\mathrm{sg}}$ is defined in Eq. (10)]. Therefore, if $T_{\mathrm{sg}} \gg T_{\mathrm{K}}$ then one expects a maximum of the Kondo contribution to the resistivity and a minimum of the weak localization correction $\Delta \sigma_{\mathrm{wl}}$ at $T \simeq T^{*}$, see Fig. 11 The positions of these extrema shift towards lower temperatures with the decreasing concentration of the magnetic impurities. At lower temperatures $T \lesssim T_{\mathrm{sg}}$ when the spin glass state is formed both

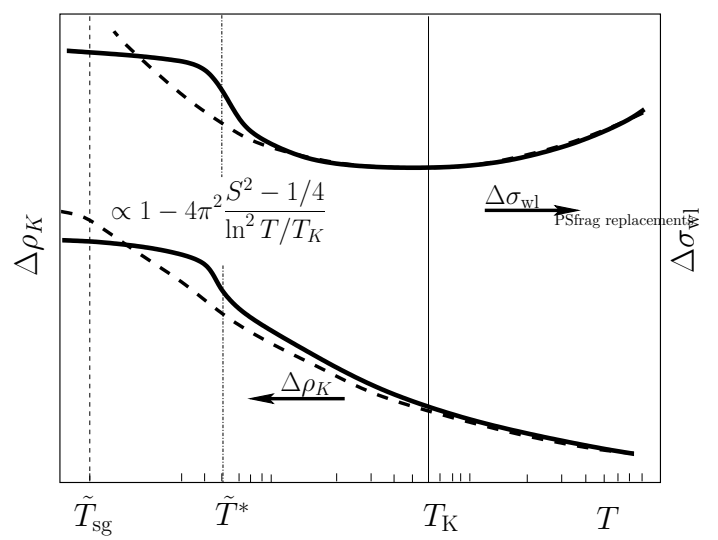

FIG. 2: Schematic picture of the Kondo contribution to the resistivity $\Delta \rho_{K}$ and of the weak localization correction to the conductivity $\Delta \sigma_{\mathrm{wl}}$ in samples with low concentration $\left(T_{\mathrm{sg}} \ll\right.$ $T_{\mathrm{K}}$ ) of magnetic impurities with spin $S>1 / 2$. The solid lines represent $\Delta \rho_{\mathrm{K}}$ and $\Delta \sigma_{\mathrm{wl}}$ for interacting impurities. Dashed lines show $\Delta \rho_{\mathrm{K}}$ and $\Delta \sigma_{\mathrm{wl}}$ as if the interaction were absent.

the resistivity and the WL correction to the conductivity saturate.

In the opposite case, $T_{\mathrm{sg}} \ll T_{\mathrm{K}}$, the momentum relaxation rate increases monotonically with the decreasing $T$, and eventually saturates at $T=0$, see the lower curve in Fig. 2 The saturation level depends on the value of impurity spin $S$. Thus the Kondo contribution to the Drude conductivity is a monotonic function of temperature. The spin-induced contribution to the phase relaxation rate, on the contrary, has a maximum at $T \sim T_{\mathrm{K}}$. If this contribution dominates over all other mechanisms of the phase relaxation, then the weak localization correction to the conductivity, $\Delta \sigma_{\mathrm{wl}}$, has a minimum at $T \sim T_{\mathrm{K}}$, see the upper curve in Fig. 22 The details of the low-temperature increase of $\Delta \sigma_{\mathrm{wl}}$ with the further reduction of temperature in the region $T \lesssim T_{\mathrm{K}}$ depend on the level of Kondo screening. In the case of full screening $(S=1 / 2)$, the phase relaxation rate vanishes at $T \rightarrow 0$, and $\Delta \sigma_{\mathrm{wl}}(T)$ diverges, see Eq. (58). If the screening is not complete $(S>1 / 2)$, then both the phase relaxation rate $1 / \tilde{\tau}_{\mathrm{s}}$ and the weak localization correction $\Delta \sigma_{\mathrm{wl}}$ saturate at some finite level, see Fig. 20 Within the simplest model of the spin-glass state accepted in Ref. 29, we find

$$
\Delta \sigma_{\mathrm{wl}}=\frac{e^{2}}{2 \pi \hbar} \sqrt{\frac{D \tilde{\tau}_{\mathrm{s}}}{2}}, \quad \frac{1}{\tilde{\tau}_{\mathrm{s}}} \simeq \frac{8 \pi n_{\mathrm{s}}}{\nu} \frac{(S-1 / 2)^{2}}{\ln ^{2} T_{\mathrm{K}} / \tilde{T}_{\mathrm{sg}}},
$$

where the temperature $\tilde{T}_{\mathrm{sg}}$ is defined in Eq. (37). It is worth noting that the saturation occurs at temperature $\sim \tilde{T}_{\mathrm{sg}}$, well below the Kondo temperature $T_{\mathrm{K}}$.

The considered limits of $T_{\mathrm{sg}} \gg T_{\mathrm{K}}$ and $T_{\mathrm{sg}} \ll T_{\mathrm{K}}$, and the conjecture of Hertz ${ }^{26}$ allow us to understand the evolution of the temperature dependence of $\Delta \rho_{K}(T)$ and $\Delta \sigma_{\mathrm{wl}}$ with the concentration of impurities $n_{\mathrm{s}}$. The position of the maximum in $\Delta \rho_{K}(T)$ shifts continuously towards $T=0$ with the decrease of $n_{\mathrm{s}}$; it reaches $T=0$ at some finite value of $n_{\mathrm{s}}$, which corresponds to $T_{\mathrm{sg}} \sim T_{\mathrm{K}}$. 
Note that such behavior occurs irrespective to the value of $S$. Formation of the spin glass with $T_{\mathrm{sg}} \ll T_{\mathrm{K}}$ at $S>1 / 2$ does not result in the finite-temperature maximum of the function $\Delta \rho_{K}$. The position of the minimum in $\Delta \sigma_{\mathrm{wl}}$ also moves to lower values of $T$ with the decrease of $n_{\mathrm{s}}$. This shift, however, stops at $T \sim T_{\mathrm{K}}$; thus the minimum occurs at a finite temperature even in the limit $T_{\mathrm{sg}} \ll T_{\mathrm{K}}$. (Once again, here we assume that $\tau_{\mathrm{s}}$ is the shortest of the phase relaxation times.)

Electron scattering off interacting magnetic impurities leads to the energy transfer from electron to the system of localized spins. The rate of collisions with a relatively large energy transfer $E$ can be calculated by means of the virial expansion. The corresponding full system of kinetic equations for the electrons and spins is derived in Section $\nabla$ The collision rate with energy loss $E$ at $|E| \gg$ $\max \left\{T_{\mathrm{sg}}, T_{\mathrm{K}}, T\right\}$ scales with $E$ as $\theta(E) T_{\mathrm{sg}} /\left(\tau_{\mathrm{s}} E^{2}\right)$. This asymptote of the rate is not sensitive to the formation of the spin glass state. However the spin glass transition affects the electron energy relaxation for smaller energy transfers, $|E| \lesssim T_{\mathrm{sg}}$.

In most part, the data of existing experimental works 5.9.10.11.30.31 can be understood within the presented here theoretical framework. The evolution of the temperature dependence of the resistivity with the concentration of magnetic impurities was studied in dilute AuFe alloys. The investigated range of the magnetic impurity $(\mathrm{Fe})$ concentration $n_{\mathrm{s}}$ covered by the data of Refs. 5 9 10, 11,30,31 is extremely broad, ranging from 3.3 ppm, see Ref. 10 , up to a few percent ${ }^{30}$. For concentrations $n_{\mathrm{s}} \gtrsim 100 \mathrm{ppm}$, the function $\Delta \rho_{K}(T)$ has a clear maximum ${ }^{5.30}$. Its position $T_{\max }$ moves to lower temperatures as the impurity concentration $n_{\mathrm{s}}$ decreases; the measured in Ref. 5 dependence of $T_{\max }$ on $n_{\mathrm{s}}$ is superlinear, in a qualitative agreement with Eq. (29). The extrapolation of the data of Ref. 5 to $T_{\max }=0$ yields the critical value of $\mathrm{Fe}$ concentration $\approx 50 \mathrm{ppm}$ for the AuFe alloy, see also Refs. 10,31. Finally, the observed ${ }^{10}$ magnetic hysteresis of the resistivity at $n_{\mathrm{s}}=7 \mathrm{ppm}$ may indicate formation of a spin glass even for such low impurity concentrations, which is possible at $S>1 / 2$, see Section IIIB 2

The weak localization correction to the conductivity in AuFe wires with low impurity concentration $\left(n_{\mathrm{s}}=7 \sim\right.$ $60 \mathrm{ppm}$ ) was studied in Refs. 10 11. There is a proper correspondence between the data ${ }^{11}$ for the sample with $n_{\mathrm{s}}=60 \mathrm{ppm}$, and the data 10 for $n_{\mathrm{s}}=10.9$ and $7.1 \mathrm{ppm}$. The values of $1 / \tau_{\mathrm{s}}$ found from the weak-localization magnetoresistance, see Eqs. (52), (59) and (75), scale roughly linearly with $n_{\mathrm{s}}$. The temperature dependence of $1 / \tau_{\mathrm{s}}$ for the investigated samples is also in accord with the theory. Namely, the phase relaxation rate exhibits a broad plateau at temperatures around $T_{\mathrm{K}} \approx 0.3 \mathrm{~K}$ (with the plateau value ${ }^{11}$ of $1 / \tau_{\mathrm{s}} \approx 6 \times 10^{10} \mathrm{~s}^{-1}$ for $\left.n_{\mathrm{s}}=60 \mathrm{ppm}\right)$. The plateau is followed by a decrease of this rate at lower temperatures. The saturation ${ }^{11}$ of $1 / \tau_{\mathrm{S}}(T)$ at $T<T_{\mathrm{K}}$ is compatible with the value of impurity spins $S>1 / 2$.

We also notice that the data of Ref. 11 for an AuFe alloy with $n_{\mathrm{s}}=15 \mathrm{ppm}$ are in sharp contrast with other experimental data5.10.11.31 and with the expectations supported by the presented theory. Indeed, Reference 11 reports the position of the Drude resistivity maximum at $T_{\max } \approx 30 \mathrm{mK}$ for the $n_{\mathrm{s}}=15 \mathrm{ppm}$ sample, which is indistinguishable from the value of $T_{\max }$ for the $n_{\mathrm{s}}=60 \mathrm{ppm}$ sample in the same work. This stability of $T_{\max }$ contradicts the dependence of $T_{\mathrm{sg}}\left(n_{\mathrm{s}}\right)$, expected from other experimental work $\mathrm{s}^{5.31}$ and from theory, see Sec. III Also, the four-fold difference of $n_{\mathrm{s}}$ between the two samples ${ }^{11}$ resulted in a 100-fold decrease of the electron phase relaxation rate $1 / \tau_{\mathrm{s}}$. This drastic change of $1 / \tau_{\mathrm{s}}$ with $n_{\mathrm{s}}$ contradicts both the quoted above measurements ${ }^{10}$ and the theoretical estimates, see Eqs. (52) and (59).

Measurements of the energy relaxation in nano-wires of $\mathrm{Au}, \mathrm{Cu}$, and $\mathrm{Ag}$ revealed the effect of individual magnetic impurities ${ }^{2}$, but there was no systematic study of the effect of RKKY interaction on the electron energy relaxation. Measurements of the relaxation rates at energy transfers $|E| \lesssim T_{\mathrm{sg}}$ may provide information about the excitations in a spin glass, but we are not aware about such measurements as of yet.

We acknowledge stimulating conversations with B.L. Altshuler. We are grateful to C. Bauerle, N. Birge, J. Mydosh, H. Pothier, L. Saminadayar for useful comments and illuminating discussions of experiments, and to C. Chamon, L. Cugliandolo, L. Ioffe for discussions of spin glass phenomena. This work was supported by NSF grants DMR97-31756, DMR01-20702, DMR0237296, and EIA02-10736.

\section{APPENDIX A: MATRIX ELEMENTS $A_{J J^{\prime}}$}

In this Appendix we present the expression for the $A_{J J^{\prime}}$ factors in Eq. (12). First we notice that the convenient form to calculate $\left\langle J M\left|\hat{S}_{\alpha}\right| J^{\prime} M^{\prime}\right\rangle$ is to use the basis of spin states for two independent spins: $\left|m_{1} m_{2}\right\rangle$, where $m_{1}$ and $m_{2}$ are the spin components along some direction. We have

$$
\begin{aligned}
\eta_{\alpha}\left(J M J^{\prime} M^{\prime}\right) & =\left\langle J M\left|\hat{S}_{\alpha}\right| J^{\prime} M^{\prime}\right\rangle \\
& =\sum_{m_{1}, m_{2}, m_{1}^{\prime}} C_{m_{1} m_{2}}^{J M}\left\langle m_{1}\left|\hat{S}_{\alpha}\right| m_{1}^{\prime}\right\rangle C_{m_{1}^{\prime} m_{2}}^{J^{\prime} M^{\prime}}
\end{aligned}
$$

where the Clebsh-Gordon coefficients are expressed in terms of the Wigner $3 j-$ symbols as

$$
C_{m_{1} m_{2}}^{J M}=\sqrt{2 J+1}\left(\begin{array}{ccc}
J & S & S \\
-M & m_{1} & m_{2}
\end{array}\right) .
$$

The matrix element $A_{J J^{\prime}}$ may be represented in terms of $\eta_{\alpha}$ :

$$
A_{J J^{\prime}}=\sum_{\alpha=x, y, z} \sum_{M^{\prime}} \eta_{\alpha}^{2}\left(J M J^{\prime} M^{\prime}\right)
$$




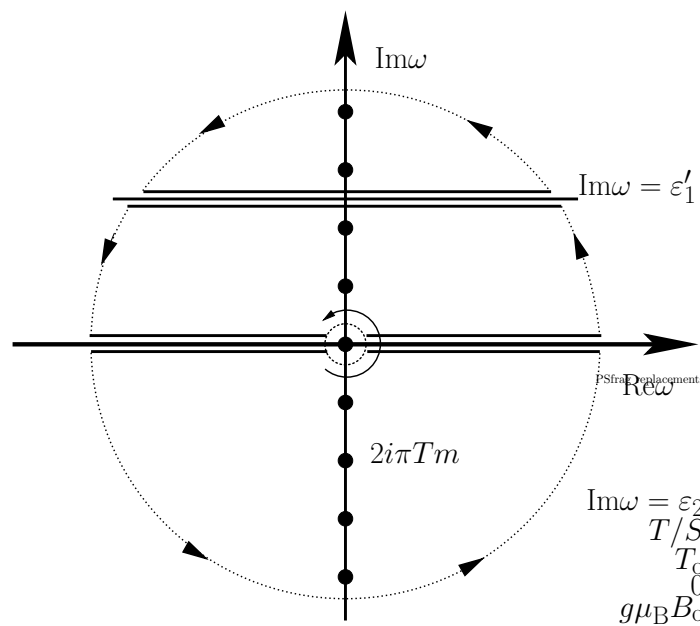

FIG. 3: The contour for calculation of the $T$-matrix, Eq. (B4), in the Matsubara representation. The contributions from the dotted parts of the contour vanish.

We find that

$$
\begin{aligned}
A_{J, J+1} & =\frac{(J+1)(2 S-J)(2 S+J+2)}{4(2 J+1)}, \\
A_{J, J} & =\frac{J(J+1)(2 J+1)}{4(2 J+1)} \\
A_{J, J-1} & =\frac{J(2 S-J+1)(2 S+J+1)}{4(2 J+1)}
\end{aligned}
$$

and all other elements vanish. From Eqs. (A3) we verify explicitly that

$$
\sum_{J, J^{\prime}=0}^{2 S}(2 J+1) A_{J J^{\prime}}=S(S+1)(2 S+1)^{2} .
$$

\section{APPENDIX B: ELECTRON T-MATRIX.}

For completeness of the presentation, we show how the imaginary part of the electron $T$-matrix may be related to the spin correlation function, defined by Eq. (3). In the Matsubara representation the $T$-matrix is given by the following expression:

$$
\mathcal{T}\left(i \varepsilon_{n}\right)=\mathcal{J}^{2} T \sum_{\omega_{m}} \int \frac{d \mathbf{p}}{(2 \pi)^{3}} \mathcal{K}\left(i \omega_{m}\right) \mathcal{G}\left(i\left(\varepsilon_{n}-\omega_{m}\right), \mathbf{p}\right),
$$

where $\mathcal{K}\left(i \omega_{m}\right)$ is the Fourier component at $\omega_{m}=2 \pi m T$ of the Matsubara spin correlator

$$
\mathcal{K}(\tau)=\sum_{\xi \xi^{\prime}} \rho_{\xi} e^{-\left(E_{\xi}-E_{\xi^{\prime}}\right) \tau}\left|\left\langle\xi|\boldsymbol{S}| \xi^{\prime}\right\rangle\right|^{2}
$$

[compare to Eqs. (3) and (4) for the real time spin correlator $K(t)$ ], and

$$
\mathcal{G}\left(i \varepsilon_{n}, \mathbf{p}\right)=\frac{1}{i \varepsilon_{n}-\xi(\mathbf{p})-\Sigma\left(\varepsilon_{n}\right)}
$$

is the electron Green's function at Matsubara frequency $\varepsilon_{n}=\pi(2 n+1) T$ [compare to Eq. (19)]. The integration over momentum in Eq. (B1) gives

$$
\mathcal{T}\left(i \varepsilon_{n}\right)=-i \pi \nu \mathcal{J}^{2} \sum_{\omega_{k}} \mathcal{K}\left(i \omega_{m}\right) \operatorname{sign}\left(\varepsilon_{n}-\omega_{m}\right) .
$$

Next we perform the standard procedure of the analytical continuation in Eq. (B2). We replace the sum over discreet $\omega_{m}$ by the integral over complex $\omega$ :

$$
T \sum_{\omega_{m}} \mathcal{F}\left(i \omega_{m}\right)=i \int_{C} \frac{d \omega}{4 \pi} \operatorname{coth} \frac{\omega}{2 T} \mathcal{F}(\omega) .
$$

This procedure is valid for an arbitrary function $\mathcal{F}$, analytic inside the contour $C$ of integration. We choose $C$ to be a circle of infinite radius with two cuts at $\operatorname{Im} \omega=0$ and $\operatorname{Im} \omega=\varepsilon_{n}$. Inside the contour of the integration, the function $\mathcal{K}(\omega)$ is analytic and only the poles of $\operatorname{coth} \omega / 2 T$ contribute to the integral. Neglecting the contribution from the pieces of the circle, and keeping the contribution along the cuts, we obtain

$$
\begin{aligned}
& \frac{\mathcal{T}\left(i \varepsilon_{n}\right)}{\pi \nu \mathcal{J}^{2}}=-\int_{-\infty}^{+\infty} \tanh \frac{\omega}{2 T} \mathcal{K}\left(i \varepsilon_{n}+\omega\right) \frac{d \omega}{2 \pi} \\
& +\operatorname{sign} \varepsilon_{n} \int_{-\infty}^{+\infty} \operatorname{coth} \frac{\omega}{2 T} \frac{\mathcal{K}(\omega+i 0)-\mathcal{K}(\omega-i 0)}{2} \frac{d \omega}{2 \pi} .
\end{aligned}
$$

The imaginary part of the $T$-matrix is given by

$$
\operatorname{Im} T(\varepsilon)=i \frac{\mathcal{T}(\varepsilon+i 0)-\mathcal{T}(\varepsilon-i 0)}{2} .
$$

We substitute Eq. (B6) in Eq. (B7), take into account relation

$$
K(\omega)=i \frac{\mathcal{K}(\omega+i 0)-\mathcal{K}(\omega-i 0)}{e^{\omega / T}-1}
$$

between the real time spin correlator, Eq. (31), and the Matsubara spin correlator, Eq. (B2), and obtain Eq. (25).

\section{APPENDIX C: COOPERON SELF ENERGY}

The Cooperon self energy $\Sigma_{\mathrm{rr}}(\cdot)$ may be obtained as a result of the analytical continuation of $\Sigma\left(\begin{array}{ll}i \varepsilon_{1}, & i \varepsilon_{1}^{\prime} \\ i \varepsilon_{2}, & i \varepsilon_{2}^{\prime}\end{array}\right)$, written in the Matsubara representation:

$$
\Sigma_{s}^{\mathrm{rr}}\left(\begin{array}{ll}
\varepsilon_{1}, & \varepsilon_{1}^{\prime} \\
\varepsilon_{2}, & \varepsilon_{2}^{\prime}
\end{array}\right)=\Sigma_{s}\left(\begin{array}{ll}
\varepsilon_{1}+i 0, & \varepsilon_{1}^{\prime}+i 0 \\
\varepsilon_{2}-i 0, & \varepsilon_{2}^{\prime}-i 0
\end{array}\right) .
$$

The Cooperon self energy is related to the scattering matrix $\mathcal{S}$ off a magnetic impurity for two electrons in a singlet state:

$$
\Sigma_{s}(\cdot)=2 \pi \nu n_{\mathrm{s}} \overline{\mathcal{S}(\cdot)} .
$$




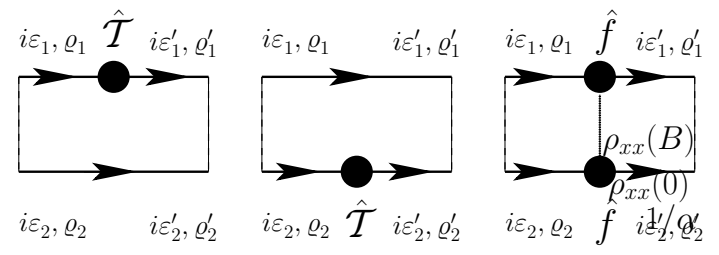

FIG. 4: The left two diagrams represent the self-energy corrections to the Cooperon and contain the single electron $T$-matrix. The diagram on the right is the vertex correction and is related to the two electron counterpart of a $T$-matrix.

In Eq. (C2) $\mathcal{S}$ is averaged over various magnetic impurities. The singlet component $\mathcal{S}_{s}$ is related to the full matrix $\hat{\mathcal{S}}$ of two electron scattering:

$$
\mathcal{S}_{s}(\cdot)=\frac{1}{2}\left(\mathcal{S}_{\downarrow \downarrow}^{\uparrow \uparrow}(\cdot)+\mathcal{S}_{\uparrow \uparrow}^{\downarrow \downarrow}(\cdot)-\mathcal{S}_{\uparrow \downarrow}^{\downarrow \uparrow}(\cdot)-\mathcal{S}_{\downarrow \uparrow}^{\uparrow \downarrow}(\cdot)\right) .
$$

Matrix $\mathcal{S}$, in its turn, contains the contribution from three diagrams, shown in Fig. 4 and has the following form:

$$
\begin{aligned}
& \mathcal{S}_{\varsigma_{2} \varsigma_{2}^{\prime} \varsigma_{1}^{\prime}}\left(\begin{array}{l}
i \varepsilon_{1}, i \varepsilon_{1}^{\prime} \\
i \varepsilon_{2}, i \varepsilon_{2}^{\prime}
\end{array}\right)=\mathcal{V}_{\varsigma_{2} \varsigma_{2}^{\prime}}^{\varsigma_{1} \varsigma_{1}^{\prime}}\left(\begin{array}{ll}
i \varepsilon_{1}, & i \varepsilon_{1}^{\prime} \\
i \varepsilon_{2}, & i \varepsilon_{2}^{\prime}
\end{array}\right) \\
& \quad-\frac{\mathcal{T}\left(i \varepsilon_{1}\right)-\mathcal{T}\left(i \varepsilon_{2}\right)}{2 \pi i \nu} \theta\left(-\varepsilon_{1} \varepsilon_{2}\right) \operatorname{sign} \varepsilon_{1} \delta_{\varepsilon_{1} \varepsilon_{1}^{\prime}} \delta_{\varepsilon_{2} \varepsilon_{2}^{\prime}} \delta_{\varsigma_{1} \varsigma_{1}^{\prime}} \delta_{\varsigma_{2} \varsigma_{2}^{\prime}} .
\end{aligned}
$$

Here the second term contains the single electron $T$-matrix considered in Appendix B Below we focus on the irreducible component of the scattering matrix of two electrons in a singlet state, represented by

$$
\begin{aligned}
& \mathcal{V}_{\varsigma_{2} \varsigma_{2}^{\prime}}^{\varsigma_{1} \varsigma_{1}^{\prime}}\left(\begin{array}{cc}
i \varepsilon_{1}, & i \varepsilon_{1}^{\prime} \\
i \varepsilon_{2}, & i \varepsilon_{2}^{\prime}
\end{array}\right)=\theta\left(-\varepsilon_{1} \varepsilon_{2}\right) \theta\left(-\varepsilon_{1}^{\prime} \varepsilon_{2}^{\prime}\right) \sum_{\xi \xi^{\prime}} \frac{e^{-E_{\xi} / T}}{Z} \\
& \quad \times T \sum_{\omega_{m}} f_{\varsigma_{1} \varsigma_{1}^{\prime}}^{\xi \xi^{\prime}}\left(i \omega_{m}\right) f_{\varsigma_{2} \varsigma_{2}^{\prime}}^{\xi \xi^{\prime}}\left(-i \omega_{m}\right) \delta_{\varepsilon_{1}+\omega_{m}, \varepsilon_{1}^{\prime}} \delta_{\varepsilon_{2}, \varepsilon_{2}^{\prime}+\omega_{m}},
\end{aligned}
$$

where $f_{\varsigma \varsigma^{\prime}}^{k k^{\prime}}\left(i \omega_{m}\right)$ is the scattering amplitude of electrons with initial spin state $\varsigma^{\prime}$ to the spin state $\varsigma$, accompanied with the change of the state of magnetic impurities from $\xi^{\prime}$ to $\xi$. The summation over $\xi$ and $\xi^{\prime}$ runs over all possible states of the impurity spin and $Z=\sum_{\xi} e^{-E_{\xi} / T}$. For scattering amplitudes in the Born approximation we have

$$
f_{\varsigma \varsigma^{\prime}}^{\xi \xi^{\prime}}=\mathcal{J}\left\langle k|\hat{\boldsymbol{S}}| k^{\prime}\right\rangle \hat{\boldsymbol{\sigma}}_{\varsigma \varsigma^{\prime}} .
$$

We substitute Eq. (C6) into Eq. (C5), and use Eq. (C3) to write down the singlet component of the vertex part of the Cooperon $\mathcal{S}$ matrix:

$$
\begin{aligned}
\mathcal{V}_{s}\left(\begin{array}{cc}
i \varepsilon_{1}, & i \varepsilon_{1}^{\prime} \\
i \varepsilon_{2}, & i \varepsilon_{2}^{\prime}
\end{array}\right)= & \mathcal{J}^{2} \theta\left(-\varepsilon_{1} \varepsilon_{2}\right) \theta\left(-\varepsilon_{1}^{\prime} \varepsilon_{2}^{\prime}\right) T \sum_{\omega_{m}} \delta \mathcal{K}_{2}\left(i \omega_{m}\right) \\
& \times \delta_{\varepsilon_{1}+\omega_{m}, \varepsilon_{1}^{\prime}} \delta_{\varepsilon_{2}, \varepsilon_{2}^{\prime}+\omega_{m} .}
\end{aligned}
$$

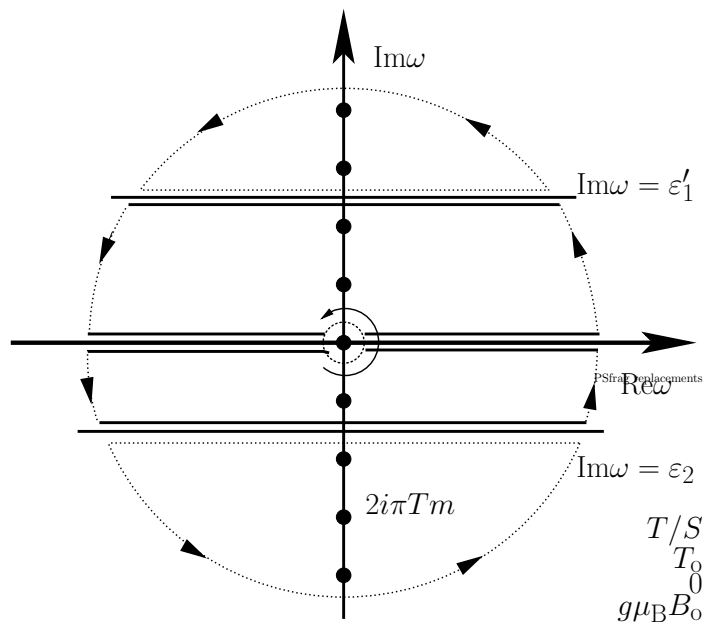

FIG. 5: The contour for calculation of the vertex correction $\hat{\mathcal{V}}_{s}$ to the Cooperon. The contributions from the dotted parts of the contour vanish.

Next we perform the analytical continuation of $\mathcal{V}_{s}$. We consider the case $\varepsilon_{1}^{\prime}>0$ and $\varepsilon_{2}<0$, see Eq. (C1). Using Eq. (B5), we replace the sum over the Matsubara frequencies, $\omega_{m}$, by the integral over a contour in the complex plane. In the present case the contour of the integration is shown in Fig. [5] and contains three cuts: $\operatorname{Im} \omega=\varepsilon_{2}, 0, \varepsilon_{1}^{\prime}$. We notice, that the contour parts above the upper cut and below the lower cut do not contribute to the integral. The remaining parts of the contour along the cuts after the continuation $i \varepsilon_{1}^{\prime} \rightarrow \varepsilon_{1}^{\prime}+i 0$ and $i \varepsilon_{2} \rightarrow \varepsilon_{2}-i 0$ give

$$
\begin{aligned}
& \mathcal{V}_{s}\left(\begin{array}{ll}
\varepsilon_{1}+i 0, & \varepsilon_{1}^{\prime}+i 0 \\
\varepsilon_{2}-i 0, & \varepsilon_{2}^{\prime}-i 0
\end{array}\right)=\int_{-\infty}^{+\infty} d \omega \delta\left(\varepsilon_{1}+\omega-\varepsilon_{1}^{\prime}\right) \delta\left(\varepsilon_{2}-\omega-\varepsilon_{2}^{\prime}\right) \\
& \quad \times 2 \pi \mathcal{J}^{2}\left\{\operatorname{coth} \frac{\omega}{2 T}\left[\delta \mathcal{K}_{2}(\omega+i 0)-\delta \mathcal{K}_{2}(\omega-i 0)\right]\right. \\
& \left.\quad-\tanh \frac{\omega}{2 T}\left[\delta \mathcal{K}_{2}\left(\omega+\varepsilon_{1}^{\prime}+i 0\right)-\delta \mathcal{K}_{2}\left(\omega+\varepsilon_{2}-i 0\right)\right]\right\} .
\end{aligned}
$$

This expression may be further simplified in the case when $\varepsilon_{1}^{\prime}=\varepsilon_{2}$ with the help of Eq. (B8).

\section{APPENDIX D: CALCULATIONS OF THE VIRIAL CORRECTION TO $\Delta \sigma_{\mathrm{wl}}$}

To calculate the WL correction to the conductivity, we substitute Eq. (49) with the self energy defined by Eq. (50) into Eq. (43). As a result we obtain

$$
\Delta \sigma_{\mathrm{wl}}=\Delta \sigma_{\mathrm{wl}}^{(0)}+\Delta \sigma_{\mathrm{wl}}^{(1, a)}+\Delta \sigma_{\mathrm{wl}}^{(1, b)}
$$


Here $\Delta \sigma_{\mathrm{wl}}^{(0)}$ is given by Eq. (45), and the second term is

$$
\begin{aligned}
\Delta \sigma_{\mathrm{wl}}^{(1, a)} & =-\frac{\Delta \sigma_{\mathrm{wl}}^{(0)}}{4} \int \frac{\delta \overline{K_{2}(\omega)}}{S(S+1)} \frac{\omega}{T} \frac{1}{1-e^{-\omega / T}} \frac{d \omega}{\pi} \\
& =\frac{\Delta \sigma_{\mathrm{wl}}^{(0)}}{2} \frac{\alpha_{S} T_{\mathrm{sg}}}{T},
\end{aligned}
$$

with $\alpha_{S}$ defined by Eq. (28), see also Table प The third term in Eq. (D1) is

$$
\Delta \sigma_{\mathrm{wl}}^{(1, b)}=-\frac{\Delta \sigma_{\mathrm{wl}}^{(0)}}{2} \int \frac{\delta \overline{K_{2}(\omega)}}{S(S+1)} \frac{\omega}{T} \frac{\varphi(\omega)}{1-e^{-\omega / T}} \frac{d \omega}{\pi},
$$

where

$$
\varphi(\omega)=\frac{1}{4}-\frac{\operatorname{Im} \sqrt{1+i \omega \tau_{\mathrm{s}} / 2}}{\omega \tau_{\mathrm{s}} \sqrt{1+\omega^{2} \tau_{\mathrm{s}}^{2} / 4}}
$$

As $\omega \tau_{\mathrm{s}} \rightarrow 0$ function $\varphi(\omega)$ vanishes. Therefore, at low temperature $T \tau_{\mathrm{s}} \ll 1$ only the second term $\Delta \sigma_{\mathrm{wl}}^{(1, a)}$ in Eq. (D1) remains. In the opposite limit, $T \tau_{\mathrm{s}} \gg 1$, we use the following property of $\varphi(\omega)$

$$
\int_{-\infty}^{+\infty} \frac{\varphi(\omega)}{\omega^{2}} d \omega=\frac{3 \pi}{32} \tau_{\mathrm{s}}
$$

and obtain

$$
\Delta \sigma_{\mathrm{wl}}^{(1, b)} \approx \frac{\pi}{120} \frac{(4 S+1)(4 S+3)}{2 S+1} \Delta \sigma_{\mathrm{wl}}^{(0)} T_{\mathrm{sg}} \tau_{\mathrm{s}}, \quad T \tau_{\mathrm{s}} \gg 1 .
$$

1 F. Pierre, A.B. Gougam, A. Anthore, H. Pothier, D. Esteve, and N.O. Birge, preprint cond-mat/0302235

${ }^{2}$ F. Pierre, H. Pothier, D. Esteve, and M.H. Devoret, Journal of Low Temp. Phys. 118, 437 (2000).

3 A. Kaminski and L.I. Glazman, Phys. Rev. Lett. 86, 2400 (2001).

4 W.J. de Haas, J.H. de Boer, and G.J. van der Berg, Physica 1, 1115 (1934).

5 O. Laborde and P. Radhakrishna, Solid State Commun. 9, 701 (1971).

6 P.J. Ford and J.A. Mydosh, Phys. Rev. B 14, 2057 (1976).

7 J.A. Mydosh, Spin Glasses (Taylor \& Francis, London, 1993).

8 U. Larsen, Phys. Rev. B 14, 4356 (1976).

9 J.S. Schilling, P.J. Ford, U. Larsen, and J.A. Mydosh, Phys. Rev. B 14, 4368 (1976).

10 P. Mohanty and R.A. Webb, Phys. Rev. Lett. 84, 4481 (2000).

11 F. Schopfer, C. Bäuerle, W. Rabaud, and L. Saminadayar, Phys. Rev. Lett. 90, 056801 (2003).

12 A.A. Bobkov, V.I. Fal'ko, and D.E. Khmel'nitskii, Zh. Exp. Teor. Fiz. 98, 703 (1990) [Sov. Phys. - JETP 71, 393 (1990)]; V.I. Fal'ko, J. Phys.: Condens. Matter 4, 3943 (1992).

13 M.G. Vavilov and L.I. Glazman, Phys. Rev. B 67, 115310 (2003).

14 G. Göppert, Y.M. Galperin, B.L. Altshuler, and H. Grabert, Phys. Rev. B 66, 195328 (2002).

15 see, e.g. A.C. Hewson, The Kondo problem to Heavy Fermions (Cambridge University Press, Cambridge, UK, 1997).

16 A.I. Larkin, and D.E. Khmelnitsky, Zh. Exp. Teor. Fiz. 58, 1789 (1970) [Sov. Phys. JETP 31, 958 (1970)].
17 A.I. Larkin, V.I. Melnikov and D.E. Khmelnitsky, Zh. Exp. Teor. Fiz. 60, 846 (1971) [Sov. Phys. JETP 33, 458 (1971)].

18 S. Hikami, A.I. Larkin and Y. Nagaoka, Progr. Theor. Phys. 63, 707 (1980).

19 B.L. Altshuler, A.G. Aronov, D.E. Khmelnitsky, A.I. Larkin in Quantum Theory of Solids (Mir publisher, Moscow, 1982); B.L. Altshuler, A.G. Aronov, M.E. Gershenson, and Yu.V. Sharvin, Sov. Sci. Rev. A.: Phys. 9, 223 (Harwood Academic Publishers, 1987).

20 V. Barzykin and I. Affleck, Phys. Rev. B 61, 6170 (2000).

21 V.M. Galitski and A.I. Larkin, Phys. Rev. B 66, 064526 (2002).

22 K.H. Fischer and J. Hertz, Spin Glasses (Cambridge University Press, New York, 1993).

23 I. Affleck and A.W.W. Ludwig, Phys. Rev. B 48, 7297 (1993).

24 P. Noziéres and A. Blandin, J. Physique 41, 193 (1980).

25 B. A. Jones and C. M. Varma, Phys. Rev. Lett. 58, 843 (1987).

26 J.A. Hertz, Phys. Rev. B 19, 4796 (1979).

27 B.L. Altshuler, A.G. Aronov, and D.E. Khmelnitsky, J. Phys. C: Solid State Phys. 15, 7367 (1982).

28 I.L. Aleiner, B.L. Altshuler, and M.E. Gershenson, Waves in Random Media 9, 201 (1999).

29 W. Wei, G. Bergmann, and R.P. Peters, Phys. Rev. B 38, 11751 (1988).

30 P.J. Ford, T.E. Whall, and J.W. Loram, Phys. Rev. B 2, 1547 (1970).

31 V. Chandrasekhar, P. Santhanam, N.A. Penebre, R.A. Webb, H. Vloeberghs, C. Van Haesendonck, and Y. Bruynseraede, Phys. Rev. Lett. 72, 2053 (1994). 